\title{
Olfactory flow in the sturgeon is externally driven
}

DOI:

10.1016/j.cbpa.2019.06.013

\section{Document Version}

Accepted author manuscript

Link to publication record in Manchester Research Explorer

\section{Citation for published version (APA):}

Garwood, R. J., Behnsen, J., Haysom, H. K., Hunt, J. N., Dalby, L. J., Quilter, S. K., Maclaine, J. S., \& Cox, J. P. L. (2019). Olfactory flow in the sturgeon is externally driven. Comparative Biochemistry and Physiology. Part A: Molecular \& Integrative Physiology . https://doi.org/10.1016/j.cbpa.2019.06.013

\section{Published in:}

Comparative Biochemistry and Physiology. Part A: Molecular \& Integrative Physiology

\section{Citing this paper}

Please note that where the full-text provided on Manchester Research Explorer is the Author Accepted Manuscript or Proof version this may differ from the final Published version. If citing, it is advised that you check and use the publisher's definitive version.

\section{General rights}

Copyright and moral rights for the publications made accessible in the Research Explorer are retained by the authors and/or other copyright owners and it is a condition of accessing publications that users recognise and abide by the legal requirements associated with these rights.

\section{Takedown policy}

If you believe that this document breaches copyright please refer to the University of Manchester's Takedown Procedures [http://man.ac.uk/04Y6Bo] or contact uml.scholarlycommunications@manchester.ac.uk providing relevant details, so we can investigate your claim.

\section{OPEN ACCESS}


3 Russell J. Garwood $^{\mathrm{a}}$, Julia Behnsen ${ }^{\mathrm{b}}$, Harriet K. Haysom ${ }^{\mathrm{c}}$, Jeremy N. Hunt ${ }^{\mathrm{d}}$, Luke J. Dalby ${ }^{\mathrm{e}}$,

4 Samuel K. Quilter ${ }^{\mathrm{e}}$, James S. Maclaine ${ }^{\mathrm{f}}$, Jonathan P. L. Cox ${ }^{\mathrm{c}^{*}}$

5

$6 \quad{ }^{a}$ School of Earth and Environmental Sciences, University of Manchester, Manchester, M13

$7 \quad 9 P L, U K$

$8{ }^{b}$ Henry Moseley X-ray Imaging Facility, University of Manchester, Manchester, M13 9PY,

$9 U K$

$10{ }^{c}$ Department of Chemistry, University of Bath, Bath, BA2 7AY, UK

$11{ }^{d}$ Jeremy Hunt Design, Unit A6, 66 Norlington Road, London, E10 6LA, UK

12 'TotalSim, Top Station Road, Brackley, NN13 7UG, UK

$13{ }^{f}$ Department of Life Sciences, Natural History Museum, Cromwell Road, London, SW7 5BD,

$14 U K$

15

16 Running title: Olfactory flow in the sturgeon

17

MS has 48 pages, 16 figures, 1 video, 1 appendix

19

Declaration of interest: None

21

$22 *$ Corresponding author

23 Dr Jonathan P.L. Cox

24 Department of Chemistry

25 University of Bath

26 Bath, BA2 7AY, UK

27 Tel. +44 1225386548

28 j.p.1.cox@bath.ac.uk 
Abstract

30 Fluid dynamics plays an important part in olfaction. Using the complementary techniques of 31 dye visualisation and computational fluid dynamics (CFD), we investigated the hydrodynamics of the nasal region of the sturgeon Huso dauricus. H. dauricus offers several experimental advantages, including a well-developed, well-supported, radial array (rosette) of visible-by-eye olfactory sensory channels. We represented these features in an anatomically accurate rigid model derived from an X-ray scan of the head of a preserved museum specimen. We validated the results from the CFD simulation by comparing them with data from the dye visualisation experiments. We found that flow through both the nasal chamber and, crucially, the sensory channels could be induced by an external flow (caused by swimming in vivo) at a physiologically relevant Reynolds number. Flow through the nasal chamber arises from the anatomical arrangement of the incurrent and excurrent nostrils, and is assisted by the broad, cartilage-supported, inner wall of the incurrent nostril. Flow through the sensory channels arises when relatively high speed flow passing through the incurrent nostril encounters the circular central support of the olfactory rosette, decelerates, and is dispersed amongst the sensory channels. Vortices within the olfactory flow may assist odorant transport to the sensory surfaces. We conclude that swimming alone is sufficient to drive olfactory flow in H. dauricus, and consider the implications of our results for the three other extant genera of sturgeons (Acipenser, Pseudoscaphirhynchus and Scaphirhynchus), and for other fishes with olfactory rosettes.

Keywords: 3D printing; Aphanopus carbo; Chimaeridae; Hexanchidae; micro-computed tomography; pressure coefficient; static pressure; streamline. 
As part of our ongoing programme to understand the fluid dynamic processes that govern the transport of odorant molecules to a fish's olfactory sensory surface (Cox, 2008; Abel et al., 2010; Holmes et al., 2011; Cox, 2013; Rygg et al., 2013; Howard et al., 2013; Agbesi et al., 2016a, 2016b), we report here the hydrodynamics of the sturgeon's nasal region. There has been no previous experimental work in this area. There has, however, been work in other areas of sturgeon olfaction (Kasumyan, 2004), e.g. on the nasal morphology (Chen and Arratia, 1994), and the olfactory epithelium (Pyatkina, 1975; Devitsyna and Kazhlayev, 1992; Zeiske et al., 2003; Hansen and Zielinski, 2005; Camacho et al., 2010).

We chose to investigate the hydrodynamics of the sturgeon's nasal region for several reasons. First, the nasal anatomy of the sturgeon (Fig. 1) suggests that flow of water through the nasal chamber may be driven by an external flow (Cox, 2008), and external flows (arising from, for example, the movement of the sturgeon as it swims forward) are relatively straightforward to replicate experimentally (Abel et al., 2010; Agbesi et al., 2016a, 2016b). Second, although we have shown that flow through the nasal chamber of a fish can be driven by an external flow (Abel et al., 2010), we have not previously investigated, in a physical model at least, the hydrodynamics in a fish's olfactory sensory channels (Fig. 1D and F, SC; a sensory channel is the region between two adjacent olfactory lamellae, highlighted by dashed lines in Fig. 1F; the olfactory epithelium is located on the surface of the olfactory lamellae). The sturgeon's well-developed radial array of olfactory lamellae ('olfactory rosette') - with distinct sensory channels that are visible through both the incurrent nostril and, in particular, the excurrent nostril (Fig. 1C-F) - afforded us an opportunity to do so. Third, because the sturgeon's olfactory lamellae appear well supported (Fig. 1C and D), they are unlikely to be deflected significantly by water flowing over or between them, and could therefore be faithfully represented by a rigid model. Finally, the olfactory hydrodynamics of the sturgeon are relevant to other fishes with olfactory rosettes, e.g. Aphanopus carbo (Holl and Meinel, 1968).

Our aim was to answer the following questions. 1) Can an external flow induce flow through the nasal chamber? 2) If so, what is the physical mechanism driving this flow? 3) Can an external flow induce flow through the sensory channels? 4) If so, what is the physical mechanism driving this flow? 
We addressed both questions by a) using dye to visualise olfactory flow in a plastic model of the sturgeon's head, and by b) simulating flow in a computational model of the sturgeon's head (computational fluid dynamics, CFD; Tu et al., 2018). For dye visualisation, the forward motion of the fish was simulated by flowing water over the static model in a flume (Agbesi et al., 2016b). We used both dye visualisation and CFD because the two techniques are complementary. Dye visualisation gives an overall impression of flow behaviour (Lim, 2000; Settles, 2005), especially around an object with a complex geometry, such as the nasal region of a fish (Abel et al., 2010; Agbesi et al., 2016a, 2016b). CFD can give the static pressure on the surface of an object, but also within a fluid, allowing one to calculate, for example, the difference in pressure between an incurrent and an excurrent nostril (Rygg et al., 2013). CFD also gives the velocity of flow, allowing one to calculate Reynolds numbers, and to visualise flow using streamlines. Streamlines may in turn help to interpret the dye visualisation experiments, to visualise flow in regions that are obscured from the observer in a physical model, and to reveal flow behaviour not apparent from the dye visualisation experiments. Finally, CFD may overcome some of the limitations of dye visualisation. But, the CFD results must be validated by a comparison with the dye visualisation results, e.g. by replicating dye behaviour with CFD-generated streamlines.

There are four extant genera of sturgeons (family Acipenseridae): Acipenser, Pseudoscaphirhynchus, Scaphirhynchus, and Huso (Nelson, 2006). The plastic model and the computational model that we used in our study were both derived from an X-ray scan of a preserved specimen of Huso dauricus, a highly active swimmer (Vecsei and Peterson, 2004) found in a variety of aquatic habitats (estuarine, riverine, coastal; Krykhtin and Svirskii, 1997). We derived the models from this particular specimen because it had the best preserved olfactory rosette of all the sturgeon specimens that we inspected. We relate our results from the models of $H$. dauricus to species from the three other genera of the Acipenseridae, and other fishes with olfactory rosettes.

\section{Materials and methods}

119 Much of the methodology has been described before (Cox, 2008; Abel et al., 2010; Holmes et 120 al., 2011; Rygg et al., 2013; Howard et al., 2013; Ramsey et al., 2015; Agbesi et al., 2016a, 121 2016b). Therefore, only essential details are given below. Further details are given in 122 Appendix A. Values for the density and dynamic viscosity of (fresh) water given in Sections $123 \quad 2.5 .2$ and 2.7 were read from Figs. 4.3 and 5.3, respectively, of Denny (1993). 


\subsection{Specimen}

126 The specimen of Huso dauricus used to construct the models is from the Natural History

127 Museum, London, UK, catalogue number BMNH 1925.8.6.2 (Fig. 1). It has a total length of $12826 \mathrm{~cm}$ (Fig. 1A, TL). Based on a) the fact that the 'mean length' of an H. dauricus yearling is $35 \mathrm{~cm}$ (Berg, 1962) and b) Fig. 5 of Koshelev et al. (2014), the specimen was likely to have

130 been less than one year old when caught, assuming that different populations of $H$. dauricus

131 (Krykhtin and Svirskii, 1997) have similar growth rates (the data of Koshelev et al. relate to

132 specimens of $H$. dauricus from the Amur estuary and lagoon; the Natural History Museum

133 specimen of $H$. dauricus is from the Amur river, precise location unknown). Since capture,

134 the specimen has been stored in $70 \%$ industrial methylated spirits, $30 \%$ distilled water.

\subsection{X-ray micro-computed tomography}

137 X-ray micro-computed tomography (micro-CT) of the head of the specimen of $H$. dauricus

138 was performed at the Henry Moseley X-ray Imaging Facility, University of Manchester, UK, 139 using an XT H 225 system (Nikon Metrology, Tring, UK) equipped with a 22 kV/225 W 140 source and a PerkinElmer XRD 1621 detector. To ensure that the olfactory lamellae were 141 fully separated from each other, as they are likely to be in vivo, the specimen was throughout the scan immersed in Fomblin Y04 (Solvay Solexis, Milan, Italy). We have used Fomblin when X-ray scanning and magnetic resonance imaging other fishes (Holmes et al., 2011;

144 Howard et al., 2013). In air, the olfactory lamellae may have adhered to one another (Abel et 145 al., 2010). The voxel size of the scan was $38 \mu \mathrm{m}$ x $38 \mu \mathrm{m}$ x $38 \mu \mathrm{m}$. The scan comprised the 146 tip of the rostrum to the gill region (Fig. 1A, box). The scan was converted into a set of 1825

147 TIFF images (e.g. Fig. 2A; [dataset] Garwood et al., 2019) using the software Drishti

148 (Version 2.6.3; Limaye, 2012).

\subsection{Surface models}

151 Surface models (Fig. 3) were generated with the software ScanIP (Synopsys, Mountain View, 152 USA). TIFF images from the micro-CT scan were imported into ScanIP. A median filter with 153 a neighbourhood radius of three pixels in the $\mathrm{x}, \mathrm{y}$ and $\mathrm{z}$ directions was applied to the TIFF images to reduce noise and to further improve the contrast between pixels corresponding to the tissue of the specimen and those corresponding to Fomblin (Fig. 2A). The TIFF images were then transformed into a mask of the head (Fig. 2C, Ma) using methodology described previously (Abel et al., 2010; Holmes et al., 2011; Howard et al., 2013; Ramsey et al., 2015;

158 Agbesi et al., 2016a, 2016b). During this process, we removed a barbel that would have obstructed flow into one of the model's nasal chambers (Fig. 3D, black area), and sealed the 
mouth (Fig. 3D, white lines). The bent rostral tip (Fig. 1A, asterisk) of the head mask was straightened with the software ZBrush 4R8 (Pixologic, Los Angeles, CA, USA), using the methodology of Agbesi et al. (2016b). The head mask was adjusted to either life-size (for CFD) or three times life-size (for dye visualisation). Surface models of the head (Fig. 3) and the regions corresponding to the plastic model's opaque and translucent parts (Fig. 3C, Op and $\mathrm{Tr}$ ) were created according to our previous methodology (e.g. Ramsey et al., 2015). The three surface models were then each exported in binary format as stereolithography (STL) files ([dataset] Haysom et al., 2019).

\subsection{Plastic model}

170 The plastic model was three times life size a) to better visualise dye behaviour in the nasal region and b) to match the Reynolds number in the dye visualisation experiments to that in vivo (Section 2.5.1). The two parts of the plastic model were 3D printed from the corresponding STL files according to the methodology of Abel et al. (2010). The opaque part (Fig. 4A) was made in off-white plastic (ASA, Stratasys, Eden Prairie, USA), giving good contrast with the red dye used to visualise flow; the translucent part (Fig. 4B-D) was made in Accura ClearVue plastic (3D Systems, Rock Hill, South Carolina, USA), facilitating dye visualisation within one of the nasal chambers. The layers arising from the $3 \mathrm{D}$ printing process were $178 \mu \mathrm{m}$ thick in the opaque part and $50 \mu \mathrm{m}$ thick in the translucent part. To minimise any possible disruption of flow in the nasal region arising from the joint between the two parts, the translucent part included the eye and the entire section of the rostrum (Fig. 4B). The two parts were glued together to give the complete model (Fig. 4). The tips of two barbels were absent from this model (Fig. 4D, circles).

\subsection{Fluid dynamics}

185

\subsubsection{Dye visualisation}

187 Dye visualisation was performed in an Eidetics Model 1520 closed-circuit, free-surface, continuous-flow flume (Wang et al., 2007) according to our previous methodology (Abel et al., 2010; Agbesi et al., 2016a, 2016b). The working section ( $\mathrm{L} \mathrm{x} \mathrm{W} \mathrm{x} \mathrm{H)} \mathrm{of} \mathrm{the} \mathrm{flume} \mathrm{was}$ $152 \mathrm{~cm}$ x $38 \mathrm{~cm} \times 51 \mathrm{~cm}$. To obtain a well-defined dye filament, we operated the flume at a free-stream speed $\left(U_{0}\right)$ of $5 \mathrm{~cm} \mathrm{~s}^{-1}$, corresponding to a Reynolds number of 500 (Section 2.7.2), indicative of laminar flow (Vogel, 1994, pp. 84-85). According to the principle of

193 dynamic similarity (Shapiro, 1961, p. 74; Vogel, 1994, p. 102), a speed of $5 \mathrm{~cm} \mathrm{~s}^{-1}$ with a

194 three times life-size model of $H$. dauricus would correspond to a swimming speed of $15 \mathrm{~cm} \mathrm{~s}^{-}$ 
${ }^{1}$ in the actual specimen, or $0.6 T L \mathrm{~s}^{-1}(T L=26 \mathrm{~cm}$; Section 2.1). Although there is no

196 information on the swimming speed of H. dauricus, it is known that sturgeon species

197 belonging to the genus Acipenser, which are morphologically similar to H. dauricus (cf. Fig.

198 1A and Fig. A.1A, Appendix A.4), can swim at speeds corresponding to $0.3-3 T L \mathrm{~s}^{-1}$ (4-

$199270 \mathrm{~cm} \mathrm{~s}^{-1}$; e.g. Peake et al., 1997; Wilga and Lauder, 1999; Deslauriers and Kieffer, 2012;

200 Cai et al., 2013; Thiem et al., 2015). Because 0.6 TL s ${ }^{-1}$ falls in this range, we considered a

201 Reynolds number of 500 to be physiologically relevant.

203 The model's pitch (Fig. 4A, Pi) was $0 \pm 5^{\circ}$; the limits of this range were similar to the values 204 observed in Acipenser transmontanus (Liao and Lauder, 2000). The yaw (Fig. 4D, Ya) was 0 $205 \pm 10^{\circ}$, similar to the yaw we observed (Section 2.8) for Acipenser ruthenus in vivo. We used 206 one roll value ( $34^{\circ}$; Fig. 4B, Rl). We visualised flow with red food dye diluted in a ratio of 207 four parts water to one part dye. The dye, as a filament, was directed at the anterior edge of 208 the head (Fig. 4A, arrowhead). The water temperature in the flume varied between $12-$ $20916.5^{\circ} \mathrm{C}$, and by no more than $2.5^{\circ} \mathrm{C}$ in a single day. Dye visualisation experiments were 210 recorded on a Panasonic HC-V500 digital camcorder (50 frames s ${ }^{-1}, 1920$ pixels x 1080 211 pixels per frame). Footage was analysed using the software Adobe Premiere Pro CC.

\subsubsection{Computational fluid dynamics}

214 The CFD simulation of olfactory flow in H. dauricus was performed on a life-sized model

215 derived from the STL model of the head (Section 2.3). A tapered extension ('tail') was added 216 to the back of the STL model using the software GeoMagic Wrap (3D Systems; Appendix 217 A.1.4.2 and A.5, Fig. A.2). We added the tail, which was just over three times the length of 218 the head, to reduce any modification to upstream flow due to the lack of a body (Abel et al., 219 2010). The STL model ([dataset] Quilter et al., 2019) was converted to a CFD mesh with the snappyHexMesh utility of the software OpenFOAM (Weller et al., 1998). The mesh comprised approximately 63 million cells and was refined in the nasal region (Fig. 5), where the prescribed cell edge length of the surface mesh was $7.8 \mu \mathrm{m}$. There were several tens of cells across the centre of each sensory channel (Fig. 5D). Adjacent to the surface of the nasal region, the mesh comprised five layers of cells, each with a prescribed thickness of $2.6 \mu \mathrm{m}$ (Fig. 5E and F), sufficient to capture the velocity gradients here. The model was placed in a computational domain of dimensions $(\mathrm{L} \mathrm{x} \mathrm{W} \mathrm{x} \mathrm{H)} 30.0 \mathrm{~m}$ x $5.6 \mathrm{~m}$ x $5.6 \mathrm{~m}$, with a velocity inlet and a pressure outlet. The model lay at the centre of the domain in the transverse plane, with the rostral tip positioned $11.9 \mathrm{~m}$ from the velocity inlet. The large size of the computational domain, together with the position of the model within it, were chosen to 
minimise flow artifacts from the walls of the domain. Pitch, yaw, and roll were $0^{\circ}$. The density and dynamic viscosity were $999.3 \mathrm{~kg} \mathrm{~m}^{-3}$ and $1.2 \times 10^{-3} \mathrm{~Pa}$ s, respectively (values for fresh water at $14{ }^{\circ} \mathrm{C}$ ). The velocity at the inlet was $15 \mathrm{~cm} \mathrm{~s}^{-1}$, to match the Reynolds number (500) for external olfactory flow in the dye visualisation experiments (Sections 2.5.1 and 2.7.2). The no slip condition was set for all solid surfaces, together with a symmetry plane (with a zero gradient of velocity and pressure across the plane) at the dorsal, ventral, and lateral surfaces of the domain. The ambient static pressure was set to 0 . Thus static pressures were gauge pressures (Massey, 1989, p. 6). Flow was assumed to be steady (based on the transient simulation described in Section 2.8), laminar (Section 2.5.1), isothermal, and incompressible. The Navier-Stokes equations governing steady laminar flow were solved with the OpenFOAM algorithm SIMPLEC, giving a field of velocity vectors. Velocities and static pressures were the averages of the last 500 iterations from a total of 2000 iterations of a converged, time-averaged solution to the Navier-Stokes equations. Convergence was checked by monitoring the volumetric flow rate through a plane of refined cells in both the incurrent and the excurrent nostril (e.g. Fig. 5C, arrowheads). Because the volumetric flow rate through this plane changed by $\leq 0.001 \%$ over the last 500 iterations of the simulation, we assumed convergence had occurred.

Results from the CFD simulations were analysed and visualised with ParaView (Ayachit, 2016). Full details are given in Appendix A.1.4.2. Static pressures were expressed as pressure coefficients (Vogel, 1988). A pressure coefficient is the ratio of the static pressure $(P)$ to the dynamic pressure (Massey, 1989, p. 98; Vogel, 1994, p. 53) of the free-stream flow $\left(1 / 2 \rho U_{0}^{2}\right)$ :

$$
C_{p}=\frac{P}{\frac{1}{2} \rho U_{0}^{2}}
$$

where $\rho$ is the density of the fluid $\left(999.3 \mathrm{~kg} \mathrm{~m}^{-3}\right.$, above); $U_{0}$ (the free-stream speed) was 15 $\mathrm{cm} \mathrm{s}^{-1}$. The difference in static pressure between the incurrent nostril and the excurrent nostril was estimated (Appendix A.1.4.2) by subtracting the average static pressure in the excurrent nostril from the point of maximum static pressure on the wall of the incurrent nostril (Fig. 6B, $\mathrm{W})$. 
Morphometric measurements were made using ParaView, Rhinoceros (Version 4.0, Robert McNeel \& Associates), and ScanIP, usually according to previous methodology (Cox, 2008; Abel et al., 2010; Holmes et al., 2011; Cox, 2013; Howard et al., 2013; Ramsey et al., 2015). The inclination of the lateral wall of the incurrent nostril was estimated from the inclination of the incurrent nostril itself to the body axis in lateral profile (Fig. 6A, $\alpha$ ). Nasal chamber volumes were estimated according to Appendix A.1.5. Morphometric measurements were made on both the left and right nasal regions.

270

\subsection{Reynolds numbers}

272

\subsubsection{General}

274 Reynolds numbers $(R e)$ for olfactory flow were calculated using either Equation 2 (Vogel, 1994, p. 85) or Equation 3 (Holmes et al., 2011):

$$
R e=\frac{U L \rho}{\mu}
$$

$$
R e=\frac{4 Q \rho}{L \mu}
$$

where $L$ is the characteristic dimension of the object, $\mu$ is the dynamic viscosity of the fluid, and $Q$ is the volumetric flow rate. Reynolds numbers in the text are given to one significant figure.

\subsubsection{Reynolds number for dye visualisation}

288 The Reynolds number for external olfactory flow (i.e. flow over the nasal region) in the 289 plastic model was calculated (Equation 2) with $U=5 \mathrm{~cm} \mathrm{~s}^{-1}$ (the free-stream speed in the 290 flume; Section 2.5.1), $L=12 \mathrm{~mm}$ (the width of the nasal region in dorsal profile, perpendicular to the direction of flow; Fig. 4C), and $\rho=998.9-999.5 \mathrm{~kg} \mathrm{~m}^{-3}$ and $\mu=1.1-$

\subsubsection{Reynolds numbers for CFD}


Calculations of Reynolds numbers for olfactory flow in the CFD simulation used $\rho=999.3 \mathrm{~kg}$ $\mathrm{m}^{-3}$ and $\mu=1.2 \times 10^{-3} \mathrm{~Pa} \mathrm{~s}$ (Section 2.5.2). Reynolds numbers for external olfactory flow were calculated (Equation 2) with $U=15 \mathrm{~cm} \mathrm{~s}^{-1}$ (the free-stream speed in the CFD simulation; Section 2.5.2) and $L=4 \mathrm{~mm}$ (Fig. 6C). To calculate Reynolds numbers for flow through the sensory channels, $U$ was measured at the centre of each sensory channel (Fig. 7B, lower inset, disk), and was found to lie in the range $0.4-20 \mathrm{~mm} \mathrm{~s}^{-1}$; $L$ was taken to be the width of the channel at this point (Fig. 7B, lower inset) and was found to lie in the range $100-460 \mu \mathrm{m}$.

302

303 Reynolds numbers for flow through each nasal chamber were calculated (Equation 3; Appendix A.1.6) with $Q=140-150 \mathrm{~mm}^{3} \mathrm{~s}^{-1}$ (the volumetric flow rate through a sagittal cross-section of the nasal chamber, indicated by line XY in Fig. 6C) and $L=13 \mathrm{~mm}$ (the wetted perimeter of that cross-section).

\subsection{Data on other sturgeon species}

Although not as extensive as the data for $H$. dauricus, we collected data on specimens from the three other extant genera of sturgeons: Acipenser schrenckii (anatomical); A. ruthenus (anatomical, behavioural); Pseudoscaphirhynchus kaufmanni (anatomical, fluid dynamic); and Scaphirhynchus platorynchus (anatomical). The (preserved) specimens of A. schrenckii $(T L=23 \mathrm{~cm}), P$. kaufmanni $(T L=52 \mathrm{~cm})$ and $S$. platorynchus $(T L=54 \mathrm{~cm})$ were from the Natural History Museum, London, UK (catalogue numbers BMNH 1925.8.6.3, 1887.4.5.17, and 1852.8.16.26, respectively; Fig. A.1, Appendix A.4). The (living, freely swimming) specimen of $A$. ruthenus $(T L \sim 65 \mathrm{~cm})$ was in a public aquarium at Bristol Zoo Gardens, UK.

The data for A. schrenckii and P. kaufmanni were derived from a micro-CT scan of each specimen (A. schrenckii in Fomblin, voxel size $38 \mu \mathrm{m}$ x $38 \mu \mathrm{m}$ x $38 \mu \mathrm{m} ;$ P. kaufmanni in air, voxel size $69 \mu \mathrm{m}$ x $69 \mu \mathrm{m}$ x $69 \mu \mathrm{m})$. Each scan was converted to a surface model. The surface model of P. kaufmanni was transformed into a plastic model for dye visualisation and a mesh for CFD. The assumption of steady flow in the CFD simulation of $H$. dauricus was based on an initial transient simulation with the P. kaufmanni CFD model. In this simulation, the static pressure at a fixed location in the fluid in the incurrent nostril was found over $\sim 3.5$ s to vary by no more than $0.07 \%$ from the equivalent steady-state simulation (Fig. A.3, Appendix A.6). We were able to collect data for external olfactory flow in the P. kaufmanni plastic and CFD models, but not for flow in the models' olfactory sensory channels, because the latter were not resolved by the X-ray scan. The surface model of A. schrenckii was not of 
sufficient quality to transform it into either a plastic or a CFD model (the contrast between

330 Fomblin and the specimen was not as good as in the $H$. dauricus X-ray scan). The data for $A$.

331

332

333

334

335

336

337

338

339

340

341

342

343

344

345

346

347

348

349

350

351

352

353

354

355

356

357

358

359

360

361

362

363

schrenckii and P. kaufmanni were collected using similar methodology to that described in the previous sections. The data for A. ruthenus and S. platorynchus were obtained by respectively observing and inspecting each specimen.

\subsection{A note on the video clips, figures, and text}

For consistency, the video clips (see Video, Supplementary data) and figures are shown in the same orientation, with the anterior part of the head or nasal region to the left. 'Left' and 'Right' labels in the figures refer to, respectively, the left and right nasal regions of the specimen of $H$. dauricus, not the models (the surface model was reflected during its preparation; Appendix A.1.2). Likewise, any reference to the left or right nasal region in the text (including figure and video clip legends) is always to the left or right nasal region of the specimen of $H$. dauricus, not the models. Copyright of the images of the specimens belongs to the Natural History Museum, London, UK.

\section{Results}

\subsection{Nasal anatomy}

The paired nasal regions of Huso dauricus are located on the dorsal surface of the head (Figs. $1 \mathrm{~B}$ and 3A-C, NR), away from the mouth, which lies on the ventral surface (Figs. 1G and 3D). Each nasal chamber (Figs. 2B and 4C, NC) is linked to the external environment by two apertures, presumed to be the incurrent nostril and the excurrent nostril (Figs. 1C-F and 6A, IN and EN). The incurrent nostril lies on the dorsoanterior surface of the head (Fig. 6A). It has a broad inner wall (Fig. 6B and C, W). The lateral part of this wall faces anteriorally (Fig. 6B; Fig. 6A and C, arrow 1) and is inclined at an angle of $60-70^{\circ}$ (Fig. 6A, $\alpha$ ). The excurrent nostril faces laterally (Fig. 6B and C, arrow 2). Its aperture is wide, with an area three times larger than the incurrent nostril. The incurrent and excurrent nostrils are separated by a thin flap, referred to here as the nasal bridge (Figs. 1E, 2B and 6A, NB).

Each nasal chamber comprises an olfactory rosette (Figs. 1C, E, 2B, 7B and C) and a relatively large space that connects the incurrent nostril to the excurrent nostril (Fig. 2B, NC). The olfactory rosette is located on the medial wall of the nasal chamber (Fig. 2B, OR), and is made up of an array of olfactory lamellae emanating radially from a circular central support (Figs. 1C, E, 6A, 7B and C, CS). When viewed anterolaterally, the aperture of the incurrent 
nostril lies directly above the central support, and is similar in size (Fig. 7A). The lamellae create the olfactory sensory channels (Figs. 1D, F, 2B and 6A, SC). There are 26 sensory channels in the left nasal chamber, and 24 in the right (Fig. 7B and C). Consequently, the nasal chambers are, when considered as a pair, mildly asymmetric. The sensory channels are $1-3 \mathrm{~mm}$ long, and, at their centres, $0.2-1.5 \mathrm{~mm}$ deep and $100-460 \mu \mathrm{m}$ wide, with the widths of the channels necessarily increasing distally from the central support (Fig. 7B and C). The variation in sensory channel length makes each olfactory rosette itself asymmetric. With the exception of two sensory channels in the right nasal chamber (Fig. 7B, upper inset), the sensory channels in the surface model were intact. Based on their physical location, and also on flow behaviour in the nasal chamber (Sections 3.2 and 3.3), the sensory channels can be divided into lateral, dorsal, medial, and ventral quadrants (Fig. 7B and C).

The olfactory lamellae are shallow, blade-like structures (Fig. 6A). The dorsal edges of two lamellae in the left nasal chamber were bent in the surface model (Fig. 7C, black disks). Since none of the other lamellae in either the left or the right nasal chamber were bent in this manner, we assume that these two lamellae had become bent well before the X-ray scan (e.g. when the specimen was captured), and not as a result of immersing the specimen in Fomblin for the X-ray scan. Secondary folds are present on the lamellae (just discernible in Fig. 1D, and highlighted by white lines in Fig. 1F) but were not resolved in the X-ray scan, and are therefore not present in the models.

\subsection{Dye visualisation}

Flow through the nasal chamber of the plastic model of $H$. dauricus was induced by an external flow (Reynolds number 500), with the head either directly facing flow, or with it pitched, or yawed. A dye filament directed at the anterior edge of the head was deflected over the dorsal surface, entered the nasal chamber via the incurrent nostril, and then exited via the excurrent nostril, confirming the roles of these two apertures (Fig. 8A-C, Video clips 1-3). In some instances the dye filament passed through the nasal chamber intact, suggesting a significant pressure difference between the incurrent and excurrent nostrils (Fig. 8D, Video clip 4).

An external flow could also induce flow through the plastic model's sensory channels. (Although the models did not contain any sensory elements, we retain the term 'sensory channels' for consistency.) A dye filament entering the nasal chamber could fan in the vicinity of the central support (Fig. 8E, Video clip 5), indicative of flow decelerating (Shapiro, 1972), and suggesting that the surface of the central support was a region of relatively high static 
pressure. The fanning behaviour could result in the passage of dye through the sensory channels in all four quadrants of the olfactory rosette (Fig. 8C and F-K, Video clips 3 and 6-11). We observed dye passing through 15 of the 26 sensory channels in the left nasal region and 11 of the 24 channels in the right nasal region (Fig. 7B and C, yellow disks). We did not observe, or were unable to confirm, dye passage through all of the dorsal sensory channels and seven medial sensory channels (channels $10-13,15,19$ and 20) in the left nasal region, and three dorsal sensory channels (channels 7 - 9) and all of the medial sensory channels in the right nasal region. We failed to do so either because it was difficult to orientate the model to see these channels, or because of the plastic model's opacity. Dye passed through the sensory channels from the central support to the periphery of the olfactory rosette (e.g. Fig. 8C).

Dye behaved in the nasal chamber according to where it entered the incurrent nostril. Dye passage through the sensory channels occurred when the filament entered the central, medial, or dorsal parts of the incurrent nostril, specifically: central incurrent nostril $\rightarrow$ lateral sensory channels (e.g. Fig. 8F, Video clip 6); dorsal incurrent nostril $\rightarrow$ dorsal sensory channels (Fig. 8K, Video clip 11); dorsomedial incurrent nostril $\rightarrow$ medial sensory channels (Fig. 8J, Video clip 10); and ventromedial incurrent nostril $\rightarrow$ ventral sensory channels (Fig. 8G, Video clip 7). Dye entering the dorsomedial or ventral parts of the incurrent nostril resulted in vortical structures (V1-3, Fig. 8L and M, Video clips 12 and 13; Lugt, 1983; Agbesi et al., 2016a; for brevity, hereafter we use the terms 'vortices' or 'vortex').

\subsection{Computational fluid dynamics}

421 Using the same Reynolds number (500) as for dye visualisation, olfactory flow in $H$. dauricus was investigated by CFD, with the head directly facing flow (pitch, yaw, and roll $0^{\circ}$ ). The results from the CFD simulation were consistent with the dye visualisation experiments, indicating that the CFD results were valid. Thus, the behaviour of dye in the plastic model in the flume could be replicated by streamlines generated from the CFD simulation (Fig. 9). For example, dye behaviour in Video clip 4 (Fig. 8D) could be replicated by the streamline shown in Fig. 9A, and vortices V1-3 in Video clips 12 and 13 (Fig. 8L and M) could be replicated by the streamlines shown in Fig. 9B and C. In addition, streamlines that passed through the sensory channels entered the incurrent nostril at similar points to the dye filament (Fig. 10A-D; Section 3.2). The CFD simulation also showed that, as suggested by the dye visualisation experiments: a) there was a significant pressure difference between the incurrent nostril and the excurrent nostril: $10 \mathrm{~Pa}, \sim 90 \%$ of the dynamic pressure of the free-stream 
relatively high static pressure (Figs. 11C and 12); and c) flow decelerated as it approached the central support (Fig. 13).

Additionally, the CFD simulation showed that: a) the model's stagnation point was located on the rostral tip (Fig. 11A, disk); b) the lateral wall of the incurrent nostril was also a region of particularly high static pressure (Fig. 11B); c) streamlines passing through the sensory channels had a relatively high speed $\left(>8 \mathrm{~cm} \mathrm{~s}^{-1}\right)$ when they passed through the incurrent nostril (Fig. 10E); d) these streamlines impinged on the region of relatively high static pressure on the olfactory rosette's central support (Fig. 12); e) vortices V1 and V2 arose from streamlines passing through the dorsal and medial sensory channels, respectively (Fig. 12B and C); f) streamlines passing through some medial and ventral sensory channels (channels 21 and 24 in the left nasal chamber, $17-21$ in the right) produced a vortex within these sensory channels (e.g. Fig. 12C, V4); g) the volumetric flow rate through the nasal chamber was $140-150 \mathrm{~mm}^{3} \mathrm{~s}^{-1}\left(\sim\right.$ two nasal chamber volumes $\mathrm{s}^{-1}$; the volume of the nasal chamber was $70 \mathrm{~mm}^{3}$ ); and h) the Reynolds number for flow through the nasal chamber was 40 . Reynolds numbers for flow through the sensory channels ranged from 0.1 to 8 .

\section{Discussion}

\subsection{Flow through the nasal chamber is induced by an external flow}

We showed that flow through the nasal chamber of a model of the sturgeon Huso dauricus can be induced by an external flow at a physiologically relevant Reynolds number (500). In vivo, the origin of this external flow would almost certainly be $H$. dauricus's own movement as it swims forward, given that it is a highly active swimmer (Vecsei and Peterson, 2004). An oncoming environmental (e.g. riverine) current could contribute further to external flow.

The pressure difference driving flow through the nasal chamber of $H$. dauricus may be attributed to three sources:

a) The distribution of static pressure on the surface of the head. The incurrent nostril is located in a region of high static pressure $(C p>0$; Fig. 11), caused by the inclined dorsoanterior surface of the head impeding flow. The lateral wall of the incurrent nostril, which faces oncoming flow (Fig. 11B), is a region of particularly high static pressure, with $C p$ rising to $>0.99$ (Fig. 11B, disk). The lateral wall is supported by a cartilaginous bar (Fig. 11B, CB), which may help it withstand the relatively high pressures to which it is exposed (Vogel, 1988). The excurrent 
nostril, on the other hand, is located on the lateral surface of the head, in a region of low static pressure $(C p<0$; Fig. 11C), caused by flow accelerating around the nasal region. As a result of the distribution of static pressure on the surface of the head, there will be a tendency for flow to be forced into the incurrent nostril and to be drawn out of the excurrent nostril. Vogel (1988) has made this point previously in respect to the nasal regions of fishes.

b) The excurrent nostril is roughly perpendicular to oncoming flow (Fig. 11B). The fluid within it should therefore experience only the ambient static pressure of the fluid. This proved to be nearly the case in the CFD simulation, where the average static pressure of the fluid in the excurrent nostril was $1 \mathrm{~Pa}$ (ambient static pressure $=0$ ), $9 \%$ of the dynamic pressure of the free

c) Viscous entrainment (Cox, 2008). Water flowing over the relatively wide excurrent nostril should entrain fluid from this nostril.

Thus, the overall pressure difference driving flow through $H$. dauricus's nasal chamber arises from the position and orientation of the incurrent and excurrent nostrils on the surface of the head, probably in conjunction with entrainment from the excurrent nostril. External flow is captured very efficiently by this anatomical arrangement ( $~ 90 \%$ of the dynamic pressure of the free stream is harnessed).

\subsection{Flow through the olfactory sensory channels is induced by an external flow} Crucially, we showed that an external flow also induced flow through the olfactory sensory channels of the $H$. dauricus plastic model. In the dye visualisation experiments, we observed flow through about half $(15 / 26)$ of the sensory channels in the left nasal region, and about half $(11 / 24)$ in the right nasal region (Fig. 7B and C). The remaining sensory channels were located in the dorsal and medial quadrants of the olfactory rosette, which were difficult to see in the plastic model. We were able, however, to infer from the CFD simulation that flow had probably occurred through some of the obscured channels. Thus CFD-generated streamlines in the obscured channels of the left nasal chamber recreated two of the vortices observed in the dye visualisation experiments (Fig. 8L, V1 and V2; Video clip 12): streamlines passing through dorsal sensory channels 7 and 8 recreated vortex V1 and streamlines passing through medial sensory channels $10-16$ recreated vortex V2 (Fig. 9B). The agreement between the CFD-generated streamlines and dye behaviour suggests that flow did in fact occur through sensory channels 7, 8, 10-13 and 15, although we did not observe it directly. In other words, 
vortices V1 and V2 in Fig. 8L/Video clip 12 are indicative of flow through seven additional sensory channels in the left nasal chamber (Fig. 7C, red disks). Therefore, dye may have passed through up to 22 of the 26 sensory channels in the left nasal chamber.

Our results suggest that flow is driven through the sensory channels by the following mechanism. Relatively high speed (and therefore high momentum) flow passing through the incurrent nostril (Fig. 10E) strikes the broad surface of the olfactory rosette's central support, decelerates, and as a result fans across the edge of the central support and into the sensory channels (Fig. 13). The central support therefore has both a structural function (supporting the olfactory lamellae) and a hydrodynamic function (dispersing flow over the olfactory sensory surface). The interaction between incurrent flow and the central support is facilitated by the position of the incurrent nostril over the central support (at least in an anterolateral view; Fig. 7A and B). This interaction is also facilitated by the wall of the incurrent nostril, which guides flow onto the central support. The arrangement of the incurrent nostril and the central support is not unlike an impingement jet for heat and mass transfer in engineering (Incropera et al., 2013, pp. 477 - 482), where the jet spreads over the surface it strikes rather than rebounding from it (Massey, 1989, p. 117). The widening olfactory sensory channels may act as diffusers, reducing energy losses (Massey, 1989, pp. 212-214). Odorant transport within the sensory channels occurs in a laminar regime, with Reynolds numbers in the range $0.1-8$ (i.e. inertial and viscous forces are finely balanced; Shapiro, 1961, p. 78), similar to Reynolds numbers in the sensory channels of other fishes (0.02 - 5; Cox, 2008; Holmes et al., 2011).

\subsection{Other mechanisms that may assist olfactory flow}

We cannot rule out the possibility that the beating cilia of the many non-sensory ciliated cells lining the sensory olfactory epithelium of a sturgeon (e.g. Fig. 2 of Camacho et al., 2010) assist olfactory flow, because our inanimate model did not take into account the action of these cells. Non-sensory ciliated cells in the olfactory epithelia of fishes have been shown either directly (Reiten et al., 2017) or indirectly (e.g. Teichmann, 1959) to generate water currents, although these cells may also propel mucus (Cox, 2013). Respiration, on the other hand, is not likely to assist olfactory flow in $H$. dauricus, because its mouth is located on the ventral surface of its body (Fig. 1G). Indeed, the movements of the mouth that we observed during normal respiration in Acipenser ruthenus in vivo were slight (Fig. 1G, asterisk), suggesting that their influence would not extend to the nasal region. 
540 We identified several vortices in the internal olfactory flow of $H$. dauricus, either in the 541 relatively large space connecting the incurrent nostril to the excurrent nostril (Fig. 8L and M, 542 V1-3), or within some of the medial and ventral sensory channels (e.g. Fig. 12C, V4). The vortices could have two beneficial effects on odorant transport. First, V1 could drive flow into

544 the lateral sensory channels (Fig. 12B, dashed line through lateral sensory channel 3), thereby assisting convective odorant transport to these channels. Similarly, V2 could draw flow out of the ventral sensory channels (Fig. 12C, dashed line through ventral sensory channel 23). Second, V4 could assist diffusive odorant transport by creating a relatively steep velocity gradient (Bashor et al., 1974; Vogel, 1994, p. 212 and pp. 355-356). The presence of vortices in the nasal chamber of $H$. dauricus supports the notion that they are not uncommon elements in ichthyic olfactory flow (Agbesi et al., 2016b). The vortices observed here, however, occurred at lower Reynolds numbers $(0.1-8$ and 40$)$ than the ones observed previously $(700-5,000$; Agbesi et al., 2016a, 2016b).

\subsection{Implications for olfactory flow in other sturgeons and other fishes}

555 The nasal anatomy of the preserved specimens from the three other extant genera of sturgeons (Acipenser, Pseudoscaphirhynchus and Scaphirhynchus) that we inspected was similar to that of $H$. dauricus. They all had: a) olfactory rosettes comprising $\sim 20-25$ sensory channels (cf. 19 - 32 sensory channels in the olfactory rosettes of other species of Acipenser and Scaphirhynchus: Fig. 1 of Pyatkina, 1975; Table 2 of Chen and Arratia, 1994; Fig. 2 of Camacho et al., 2010); b) similar narial arrangements (this was also true of A. ruthenus in vivo), with the incurrent nostril on the dorsoanterior surface of the nasal region, and the excurrent nostril on the lateral surface of the head, roughly perpendicular to oncoming flow (Figs. 14 and 15); and c) an incurrent nostril with a broad inner wall, the lateral part of which faced anteriorally (Figs. 14C, 15C and D). Furthermore, inspection of the X-ray scans of Acipenser schrenckii and Pseudoscaphirhynchus kaufmanni showed that, as in H. dauricus, the lateral part of the incurrent nostril wall in these two species is supported by a cartilaginous bar. We were able to visualise the cartilaginous bar in situ in the surface model of P. kaufmanni (Fig. 14C, CB). Also as in H. dauricus, the incurrent nostril of both A. schrenckii and P. kaufmanni is positioned over the olfactory rosette's central support in an anterolateral view (Figs. 14C, inset, and 15B). Finally, like H. dauricus, the number of sensory channels in A. schrenckii was, as determined from the surface model, mildly asymmetric (26 in the left nasal chamber, 24 in the right), so such asymmetry may be common in sturgeons. 
574 Given these anatomical similarities, we expect olfactory flow in other sturgeons to be very

575 similar to that in the $H$. dauricus models, with external flow driving flow through the nasal 576 chamber and olfactory sensory channels, and the presence of vortices in the nasal chamber.

577 Indeed, we have shown in a plastic model of $P$. kaufmanni that flow through the nasal chamber 578 is driven by an external flow (Video clip 14), and in both a plastic and a CFD model of $P$. 579 kaufmanni that vortices did occur in the nasal chamber (Fig. 16; Video clip 15). The externally580 induced olfactory flow and the vortices in the P. kaufmanni models both occurred at Reynolds 581 numbers (700 and 20, respectively) similar to those in the H. dauricus models (500 and 40, 582 respectively). We were unable, however, to determine whether external flow can drive flow 583 through the olfactory sensory channels of $P$. kaufmanni, because these channels were not 584 resolved in the plastic or the CFD model of this specimen.

586 We observed two variable features in the nasal anatomy of the sturgeon. The first was the inclination of the lateral wall of the incurrent nostril (Fig. 6A, $\alpha$ ). In Acipenser and Huso, the inclination was quite steep $\left(\alpha=50-75^{\circ}\right)$. In Pseudoscaphirhynchus and Scaphirhynchus, which have flattened rostra (e.g. Fig. 14B), the inclination was less $\left(\alpha=35-50^{\circ}\right)$. We would expect external flow to be captured more efficiently at greater inclinations, because the obstruction to flow will be greater, and therefore the pressure coefficient on the incurrent nostril wall will be greater. This did indeed seem to be true in the CFD simulations for $H$. dauricus and $P$. kaufmanni $(90 \%$ of the dynamic pressure available from the external flow is harnessed in H. dauricus v. $70-80 \%$ in P. kaufmanni). The second variable anatomical feature was the anterior edge of the excurrent nostril, which was raised in A. schrenckii (Fig. 15A-C), but not in the other specimens. The raised edge should, by acting as a bluff body, reduce further the static pressure at the excurrent nostril (Douglas et al., 1985, p. 296), and therefore lead to more efficient capture of external flow.

600 Other fishes apart from sturgeons have well-developed olfactory rosettes, including Aphanopus carbo (Trichiuridae; Holl and Meinel, 1968), chimaeras (Chimaeridae; Howard et al., 2013), and cow sharks (Hexanchidae; Meng and Yin, 1981; Ferrando et al., 2017). The number of sensory channels in the olfactory rosettes of these fishes $(18-36)$ is similar to that in sturgeons.

604 In addition, like sturgeons, the incurrent nostrils of Aphanopus carbo and chimaeras are known 605 to be situated over the olfactory rosette's central support (Fig. 2 of Holl and Meinel, 1968; Fig. 60611 of Howard et al., 2013). Furthermore, the relatively long incurrent channel of chimaeras, together with its nozzle-like exit, appears set up to direct a stream of fluid onto the central support (Fig. 9F and G of Howard et al., 2013). Flow through the nasal chambers of these fishes 
is, however, generated by different (inferred) pumps: accessory sacs (Aphanopus carbo);

610 respiration (chimaeras); and swimming (cow sharks). But because these fishes have olfactory

611 rosettes that are similar to those of sturgeons, we suggest that the different pumps all perform

612 the same function. Thus each type of pump is likely to drive flow onto the central support,

613 where it then decelerates and is dispersed amongst the sensory channels. In other words, it is

614 likely that flow can be driven through the sensory channels of an olfactory rosette by the action

615 of a single pump, although the nature of that pump (accessory sacs, respiration, swimming) can

616 vary.

617

618

\subsection{Limitations}

619 The dye visualisation experiments involving the plastic model of $H$. dauricus were limited in several ways. a) The model lacked a body. The resultant abrupt change in geometry at the back of the head caused flow to separate at this point (Video clip 16), which may have modified upstream flow (e.g. in the nasal region). b) The model bears fine ridges (178 $\mu \mathrm{m}$ thick in the opaque part and $50 \mu \mathrm{m}$ thick in the translucent part) arising from the 3D printing process, which builds the models in layers (Section 2.4). c) The edge of the excurrent nostril in the opaque nasal region is not as smooth as it is in the specimen (cf. Video clip 5 and Fig. 1D), another consequence of the resolution of the 3D printing process. Because the model was three times life-size, its effective resolution was three times the resolution of the X-ray scan $(38 \mu \mathrm{m})$, i.e. $114 \mu \mathrm{m}$. Thus the $178 \mu \mathrm{m}$ thick ridges in the model's opaque part would have lost some of the detail of the X-ray scan. d) The walls of the flume could have affected olfactory flow in the model.

632 Each of the above limitations was addressed by the CFD simulation, where: a) the model bore 633 a tapered extension to prevent flow separating from its posterior surface (Fig. A.2, Appendix 634 A.5); b) the surface of the model was smooth; c) the edge of the excurrent nostril was faithfully reproduced (Fig. 5A); d) the distance between the model and the walls of the computational domain was substantial $(\sim 2.8 \mathrm{~m}$; in the flume it was $\geq 14 \mathrm{~cm})$. Given that olfactory flow in the dye visualisation experiments was similar to that in the CFD simulation, we conclude that the

638 limitations of the dye visualisation experiments did not significantly affect olfactory flow in the plastic model.

There were several limitations that we did not address in the $H$. dauricus models. a) We assumed that the sturgeon's olfactory lamellae were unlikely to be deflected by water flowing 
644 region of $A$. ruthenus in vivo, the continuous movement of the fish meant that we were unable

645 to do so. b) Whilst the secondary folds were present in the preserved specimen (Fig. 1D and $646 \mathrm{~F})$, they were not resolved in the X-ray scan of $H$. dauricus, and so were not present in our 647 models. We could not therefore observe their hydrodynamic effect. A higher resolution micro648 CT scan may in the future allow one to do so, e.g. by CFD. c) There were two small gaps 649 between the olfactory lamellae and the central support in the right nasal chamber (Fig. 7B, 650 upper inset, disks). d) The dorsal edges of two olfactory lamellae in the left nasal region were 651 bent (Fig. 7C, black disks). e) Prior to the X-ray scan, the H. dauricus specimen is likely to 652 have undergone some shrinkage during its ( 90 years) period of storage in alcoholic 653 preservative (Abel et al., 2010). f) In vivo, as we observed in A. ruthenus, the eyes of a sturgeon are convex. The eyes of the preserved specimen of $H$. dauricus used to construct our models had, however, collapsed to a flattened state (similar to Fig. 15B). g) The barbels in the models were either truncated or flattened against the body (Figs. 3D and 4D). In vivo, based on our aquarium observations of $A$. ruthenus, the barbels are likely to be extended ventrally (Fig. 1G). h) Flow was steady in our experiments. In vivo, flow is likely to be both steady (e.g. when the sturgeon is cruising) and unsteady (e.g. when the sturgeon is turning). i) We did not investigate the effect the substrate has on olfactory flow. In our experiments, the ventral surface of each model was placed well away from the substrate. In vivo, sturgeons typically swim close to the substrate (Vecsei and Peterson, 2004). The aquarium specimen of $A$. ruthenus could, however, swim well above the substrate. H. dauricus, as a highly active swimmer (Vecsei and Peterson, 2004), may also swim well above the substrate.

666 Although some of these limitations may have modified olfactory flow, given the sensory channels were generally fully formed, and the nasal anatomy of $H$. dauricus was very similar to that of $A$. ruthenus in vivo, the extent of the modification is unlikely to have been great.

669 Consequently, we believe that olfactory flow in the models of $H$. dauricus is a fairly accurate reflection of olfactory flow in vivo.

\section{Conclusion}

673 An external flow alone is sufficient to drive water through both the nasal chamber and the olfactory sensory channels of a sturgeon. The arrangement of the incurrent and excurrent nostrils on the surface of the head causes the pressure difference that drives flow through the nasal chamber, whilst the central support of the olfactory rosette is responsible for dispersing flow amongst the sensory channels. The wall of the incurrent nostril is instrumental in both 
678 processes. This study is we believe the first physical demonstration of externally induced

679 flow through the olfactory sensory channels of a fish.

680

681 Acknowledgements

682 We thank Laser Lines/Ogle Models and Prototypes for 3D printing, Matt Cross, Paul Frith, 683 Mary Mahon, George Oates, Ian Trussler, Zhijin Wang, and Simon Wharf for technical 684 assistance, Ismet Gursul and Richie Gill for allowing us to use, respectively, the flume and 685 ScanIP software in the Department of Mechanical Engineering at Bath, Kevin Webb and Nic 686 Delves-Broughton for photography, David Cleaver for helpful discussions, Dan Sykes and 687 Mawuli Agbesi for contributing to the work on Pseudoscaphirhynchus kaufmanni (X-ray scan 688 and dye visualisation, respectively), Xavier Mear for German to English translation, and Olivia 689 Edgar for facilitating the observations of Acipenser ruthenus at Bristol Zoo Gardens, UK.

690

$691 \quad$ Funding statement

692 This work was supported by the University of Bath's Alumni Fund. The Engineering and 693 Physical Sciences Research Council, UK, funded the Henry Moseley X-ray Imaging Facility 694 through the Royce Institute for Advanced Materials (grants EP/F007906/1, EP/F001452/1, 695 EP/I02249X, EP/M010619/1, EP/F028431/1, EP/M022498/1 and EP/R00661X/1). The 696 funding sources had no involvement in the conduct of the research, or the preparation of the 697 article. 
699 Abel, R.L., Maclaine, J.S., Cotton, R., Xuan, V.B., Nickels, T.B., Clark, T.H., Wang, Z.,

700 Cox, J.P.L., 2010. Functional morphology of the nasal region of a hammerhead shark. Comp.

701 Biochem. Physiol. A 155, 464-475.

702 Agbesi, M.P.K., Naylor, S., Perkins, E., Borsuk, H.S., Sykes, D., Maclaine, J.S., Wang, Z.,

703 Cox, J.P.L., 2016a. Complex flow in the nasal region of guitarfishes. Comp. Biochem.

704 Physiol. A 193, 52-63.

705 Agbesi, M.P.K., Borsuk, H.S., Hunt, J.N., Maclaine, J.S., Abel, R.L., Sykes, D., Ramsey

706 A.T., Wang, Z., Cox, J.P.L., 2016b. Motion-driven flow in an unusual piscine nasal region.

707 Zoology 119, 500-510.

708 Ayachit, U., 2016. The ParaView Guide. Kitware Inc.

709 Bashor, D.P., Beuerman, R.W., Easton, D.M., 1974. Ciliary action and normal movement of 710 odorant wavefronts in garfish nasal capsule of Lepisosteus osseus. Experientia, 30, 777-779.

711 Berg, L.S., 1962. Freshwater fishes of the U.S.S.R and adjacent countries. Israel Program for 712 Scientific Translations, Jerusalem.

713 Cai, L., Taupier, R., Johnson, D., Tu, Z., Liu, G., Huang, Y., 2013. Swimming capability and 714 swimming behavior of juvenile Acipenser schrenckii. J. Exp. Zool. 319A, 149-155.

715 Camacho, S., Ostos-Garrido, M.V., Domezain, A., Carmona, R., 2010. Study of the olfactory 716 epithelium in the developing sturgeon. Characterization of the crypt cells. Chem. Senses. 35, $717 \quad 147-156$.

718 Chen, X.-Y., Arratia, G., 1994. Olfactory organ of Acipenseriformes and comparison with 719 other Actinopterygians. J. Morph. 222, 241-267.

720 Cox, J.P.L., 2008. Hydrodynamic aspects of fish olfaction. J. Roy. Soc. Interface 5, 575-593.

721 Cox, J.P.L., 2013. Ciliary function in the olfactory organs of sharks and rays. Fish and Fish. 722 14, 364-390.

723 Denny, M.W., 1993. Air and Water. Princeton University Press, Princeton.

724 Deslauriers, D., Kieffer, J.D., 2012. The effects of temperature on swimming performance of 725 juvenile shortnose sturgeon (Acipenser brevirostrum). J. Appl. Ichthyol. 28, 176-181. 
726 Devitsyna, G.V., Kazhlayev, A.A., 1992. Development of chemosensory organs in the

727 Siberian sturgeon Acipeser baerii and stellate sturgeon A. stellatus. Vopr. Ikhtiol. 32, 167-

728175.

729 Douglas, J.F., Gasiorek, J.M., Swaffield, J.A., 1985. Fluid Mechanics. 2nd ed. Pitman,

730 Massachusetts.

731 Ferrando, S., Gallus, L., Amaroli, A., Gambardella, C., Waryani, B., Di Blasi, D., Vacchi,

732 M., 2017. Gross anatomy and histology of the olfactory rosette of the shark Heptranchias

733 perlo. Zoology 122, 27-37.

734 Garwood, R.J., Behnsen, J., Maclaine, J.S., Cox, J.P.L., 2019. TIFF images from X-ray scan of

735 Huso dauricus. Mendeley Data. (Final link not yet available.)

736 Hansen, A., Zielinski, B.S., 2005. Diversity in the olfactory epithelium of bony fishes:

737 development, lamellar arrangement, sensory neuron types and transduction components. J.

738 Neurocytol., 34, 183-208.

739 Haysom, H.K., Hunt, J.N., Cox, J.P.L., 2019. STL model of head of Huso dauricus.

740 Mendeley Data. (Final link not yet available.)

741 Holl, A., Meinel, W., 1968. The olfactory organ of the deep-sea fish Aphanopus carbo

742 (Percomorphi, Trichiuridae). Helgoländer wiss. Meeresunters. 18, 404-423. In German, with

743 an English summary.

744 Holmes, W.M., Cotton, R., Xuan, V.B., Rygg, A.D., Craven, B.A., Abel, R.L., Slack, R.,

745 Cox, J.P.L., 2011. Three-dimensional structure of the nasal passageway of a hagfish and its

746 implications for olfaction. Anat. Rec. 294, 1045-1056.

747 Howard, L.E., Holmes, W.M., Ferrando, S., Maclaine, J.S., Kelsh, R.N., Ramsey, A., Abel,

748 R.L., Cox, J.P.L., 2013. Functional nasal morphology of chimaerid fishes. J. Morph. 274,

749 987-1009.

750 Incropera. F.P., Dewitt, D.P., Bergman, T.L., Lavine, A.S., 2013. Principles of Heat and

751 Mass Transfer. 7th ed. John Wiley \& Sons, Singapore.

752 Kasumyan, A.O., 2004. The olfactory system in fish: structure, function, and role in behavior.

753 J. Ichthyol. 44, S180-S223.

754 Koshelev, V.N., Mikheev, P.B., Shmigirilov, A.P., 2014. Age and growth of kaluga

755 Acipenser dauricus from the estuary of the Amur and its lagoon. J. Ichthyol. 54, 165-176. 

dauricus, and Amur sturgeon, Acipenser schrenckii. Environ. Biol. Fishes 48, 231-239.

758

759

760

761

762

763

764

765

766

767

768

769

770

771

772

773

774

775

776

777

778

779

780

781

782

783

784

Liao, J., Lauder, G.V., 2000. Function of the heterocercal tail in white sturgeon: flow visualization during steady swimming and vertical maneuvering. J. Exp. Biol. 203, 35853594.

Lim, T.T., 2000. Dye and smoke visualization. In: Smits, A.J., Lim, T.T. (Eds.), Flow Visualization. Imperial College Press, London, pp. 43-72.

Limaye, A., 2012. Drishti: a volume exploration and presentation tool. In: Stock, S.R. (Ed.), Proceedings SPIE 8506, Developments in X-ray Tomography VIII, 85060X.

Lugt, H.J., 1983. Vortex Flow in Nature and Technology. John Wiley \& Sons, New York. Massey, B.S., 1989. Mechanics of Fluids, 6th ed. Van Nostrand Reinhold, London.

Meng, Q., Yin, M., 1981. A study of the olfactory organ of the sharks. Trans. Chinese Ichthyol. Soc. 2, 1-24. In Chinese, with an English summary.

Nelson, J.S., 2006. Fishes of the World. 4th ed. John Wiley \& Sons, New Jersey. Peake, S., Beamish, F.W.H., McKinley, R.S., Scruton, D.A., Katopodis, C., 1997. Relating swimming performance of lake sturgeon, Acipenser fulvescens, to fishway design. Can. J. Fish. Aquat. Sci. 54, 1361-1366.

Pyatkina, G.A., 1975. Electron microscopic study of the olfactory organ in the sterlet Acipenser ruthenus. Arch. Anat. Gistol. Embriol. 68, 85-93. In Russian, with an English summary.

Quilter, S.K., Dalby, L.J., Cox, J.P.L., 2019. STL model of head of Huso dauricus, with tapered extension. Mendeley Data. (Final link not yet available.)

Ramsey, A., Houston, T.F., Ball, A.D., Goral, T., Barclay, M.V.L., Cox, J.P.L., 2015.

Towards an understanding of molecule capture by the antennae of male beetles belonging to the genus Rhipicera (Coleoptera, Rhipiceridae). Anat. Rec. 298, 1519-1534.

Reiten, I., Uslu, F.E., Fore, S., Pelgrims, R., Ringers, C., Verdugo, C.D., Hoffman, M., Lal, P., Kawakami, K., Pekkan, K., Yaksi, E., Jurisch-Yaksi, N., 2017. Motile-cilia-mediated flow improves sensitivity and temporal resolution of olfactory computations. Curr. Biol. 27, 166174. 
Rygg, A.D., Cox, J.P.L., Abel, R., Webb, A.G., Smith, N.B., Craven, B.A., 2013. A computational study of the hydrodynamics in the nasal region of a hammerhead shark (Sphyrna tudes): implications for olfaction. PLOS ONE 8, e59783, 1-19.

788

Settles, G.S., 2005. Sniffers: fluid-dynamic sampling for olfactory trace detection in Nature and homeland security. J. Fluids Eng. 127, 189-218.

Shapiro, A.H., 1961. Shape and Flow. Heinemann, London.

Shapiro, A.H., 1972. Pressure fields and fluid acceleration. In: National Committee for Fluid Mechanics Films, Illustrated Experiments in Fluid Mechanics. MIT Press, London, pp 39-46.

Teichmann, H., 1959. Über die Leistung des Geruchssinnes beim Aal [Anguilla anguilla (L.)] (On the performance of the sense of smell of the eel). Z. vgl. Physiol. 42, 206-254. In German.

Thiem, J.D., Dawson, J.W., Gleiss, A.C., Martins, E.G., Haro, A., Castro-Santos, T., Danylchuk, A.J., Wilson, R.P., Cooke, S.J., 2015. Accelerometer-derived activity correlates with volitional swimming speed in lake sturgeon (Acipenser fulvescens). Can. J. Zool. 93, 645-654.

799

Tu, J., Yeoh, G.-H., Liu, C., 2018. Computational Fluid Dynamics. 3rd ed. ButterworthHeinemann, Oxford.

Vecsei, P., Peterson, D., 2004. Sturgeon ecomorphology: a descriptive approach. In: LeBreton, G.T.O., Beamish, F.W.H., McKinley, R.S. (Eds.), Sturgeons and Paddlefish of

803 North America. Kluwer Academic Publishers, Dordrecht, pp. 103-133.

804 Vogel, S., 1988. How organisms use flow-induced pressures. Am. Sci. 76, 28-34. aerodynamics. J. Aircraft 44, 1877-1888.

808 Weller, H.G., Tabor, G., Jasak, H., Fureby, C., 1998. A tensorial approach to computational 809 continuum mechanics using object-oriented techniques. Comp. Phys. 12, 620-631.

810 Wilga C.D., Lauder, G.V., 1999. Locomotion in sturgeon: function of the pectoral fins. J. 811 Exp. Biol. 202, 2413-2432.

812 Zeiske, E., Kasumyan, A., Bartsch, P., Hansen, A., 2003. Early development of the olfactory 813 organ in sturgeons of the genus Acipenser: a comparative and electron microscopic study.

814 Anat. Embryol. 206, 357-372. 


\section{Figure legends}

816 Fig. 1 Specimen of Huso dauricus (BMNH 1925.8.6.2) used to generate the models for fluid 817 dynamics. (A) Right side of specimen (photograph flipped horizontally). Box: extent of X-ray 818 scan. Asterisk: bent rostral tip. (B) Lateroventral aspect of head. (C) and (E): Left nasal region. 819 (D) and (F): right nasal region. Important nasal features are highlighted by continuous or 820 dashed lines in (E) and (F). (G) Ventral aspect of head and outline of lateral aspect of head 821 showing extent of mouth opening during regular respiration (asterisk) and typical position of 822 barbels when swimming, based on our observations of Acipenser ruthenus in vivo. Image 823 flipped vertically, to match Figs. 3D and 4D. Ba: Barbel; CS: central support; EN: excurrent nostril; Ey: eye; He: head; IN: incurrent nostril; La: olfactory lamella; Mo: mouth; NB: nasal bridge; NR: nasal region; SC: sensory channel; SF: secondary fold; $T L$ : total length.

Fig. 2 Image processing. (A) TIFF image from micro-CT scan of Huso dauricus. Transverse cross-section through head. Inset: right lateral aspect of head of specimen, indicating location of cross-section (dashed vertical line). Inset image flipped horizontally. (B) Same image after application of median filter. Cartilaginous bars, nasal chamber wall, olfactory rosette, and a sensory channel highlighted in yellow. (C) Same image as (B) with mask superimposed. Scale bar applies to all three images. Ba: Barbel; Ca: cartilage; CB: cartilaginous bar; d: dorsal; Fo: Fomblin; IN: incurrent nostril; La: olfactory lamella; Ma: mask; NB: nasal bridge; NC: nasal chamber; OR: olfactory rosette; SC: sensory channel; Ti: tissue; v: ventral; W: incurrent nostril wall.

837 Fig. 3 Surface model of Huso dauricus. (A) Lateral aspect. (B) Anterior and anterolateral aspects. (C) Dorsal aspect. (D) Ventral aspect. White lines: filled regions. Black area: base of removed barbel. White areas: barbel tips absent in plastic model. Ba: Barbel; EN: excurrent nostril; IN: incurrent nostril; Mo: mouth; NR: nasal region; Op: opaque and Tr: translucent parts in plastic model, respectively.

843 Fig. 4 Plastic model of Huso dauricus. (A) Lateral aspect of opaque nasal region. (B) Lateral 844 aspect of translucent nasal region (image flipped horizontally). X, Y and Z: faces of flume. Eyes and arrows indicate observer's viewpoint. Circular symbol indicates free-stream flow towards reader. I, II, III: model orientations in flume (upright, upside down, and rolled, respectively). (C) Dorsal aspect. Box: detail of opaque nasal region. Asterisk: hole for fixing model upright or upside down in flume. (D) Ventral aspect. Circles: missing barbel tips. White area: base of removed barbel. Lines: filled regions. Images above model in $(A)-(C)$ : posterior 
region of model, highlighting aluminium peg. Arrowhead in (A): area at which dye filament directed. Scale bar in (A) applies to all images. Ba: Barbel; EN: excurrent nostril; Ey: eye; FW: outline of flume walls (not to scale); IN: incurrent nostril; $L$ : characteristic dimension of nasal region; Mo: mouth; NC: nasal chamber; Op: opaque part; Pe: aluminium peg; Pi: pitch; Rl: roll; Tr: translucent part; Ya: yaw.

Fig. 5 CFD mesh of Huso dauricus. (A) Refinement of mesh on model surface, corresponding to right nasal region of specimen. Large circles: enlargements of points (small circles) at three stages of refinement, with refinement increasing right to left. (B) Mesh representing fluid surrounding cross-section of model head (same cross-section as in Fig. 2). (C) Cross-section through right nasal region. Arrowheads: plane of refined cells for monitoring volumetric flow rate through incurrent nostril. Disks: edge of incurrent nostril. (D) Cross-section through an olfactory sensory channel. (E) Mesh representing fluid adjacent to olfactory lamellar surface. (F) The five layers of refined cells (shaded) immediately adjacent to lamellar surface. (E) and (F) redrawn for clarity. Labels in (A) and scale bars in (A) - (E) deliberately omitted to allow reader to see mesh. d: Dorsal; La: olfactory lamella; v: ventral.

Fig. 6 Nasal region of Huso dauricus surface model (corresponding to the right nasal region of specimen). (A) Lateral aspect (box, Fig. 3A). $\alpha$ : angle at which lateral wall of incurrent nostril inclined to body axis. (B) Anterior aspect (left box, Fig. 3B). (C) Dorsal aspect (box, Fig. 3C). Arrow 1 indicates lateral wall of incurrent nostril faces anteriorally, arrow 2 that excurrent nostril faces laterally. CS: Central support; EN: excurrent nostril; IN: incurrent nostril; $L$ : characteristic dimension of nasal region; La: olfactory lamella; NB: nasal bridge; SC: sensory channel (extent indicated by dashed lines); W (and dashed line): incurrent nostril wall; XY: extent of sagittal cross-section used to calculate Reynolds number for flow through the nasal chamber (Section 2.7.3).

Fig. 7 Olfactory rosettes of Huso dauricus surface model. (A) Anterolateral aspect of right nasal region (right box, Fig. 3B). (B) Cut-away of (A) to show olfactory rosette. Sensory channels marking quadrant limits of olfactory rosette are numbered. Dashed line: position of incurrent nostril. Upper inset: two gaps (disks) between olfactory lamellae and central support. Gap on left connects sensory channels 10 and 11, gap on right sensory channels 7 and 8. Lower inset: cross-section through sensory channel marked by asterisk/small curved lines in main image. (C) Olfactory rosette of left nasal region. White frames in (B) and (C) indicate the lateral (Lt), dorsal (Do), medial (Me), and ventral (Ve) quadrants of rosette. Yellow and red disks: 
channels through which dye passage observed or inferred, respectively. Black disks: bent olfactory lamellae. a: Anterior; CS: central support; d: dorsal; EN: excurrent nostril; Ey: eye; IN: incurrent nostril; $L$ : characteristic dimension of sensory channel; La: olfactory lamella; $\mathrm{p}$ : posterior; SC: sensory channel; v: ventral; W: incurrent nostril wall.

890 Fig. 8 Schematics of olfactory flow in the plastic model of Huso dauricus. The plastic model 891 is represented by the surface model. Panels (A) - (M) correspond to Video clips 1-13, 892 respectively. The surface model orientation in each panel is based on a screenshot from the 893 respective video clip. Insets in $(\mathrm{C}),(\mathrm{F})$ - $(\mathrm{K})$ show which channels (numbered) dye passes 894 through in the corresponding video clip. (N) Reference image. (A), (C) - (M): lateral views. 895 (B) Dorsal view. Large white arrows in (A), (B) and (N): direction of free-stream flow. Other 896 arrows indicate behaviour of dye. Scale bar in (N) also applies to (C) - (M). Lt, Do, Me and 897 Ve: lateral, dorsal, medial and ventral quadrants of olfactory rosette, respectively. CS: Central 898 support; EN: excurrent nostril; Ey: eye; IN: incurrent nostril; NR: nasal region; V1-3: vortices. 899

900 Fig. 9 Correspondence of CFD-generated streamlines to olfactory flow in the plastic model of 901 Huso dauricus. Streamline(s) (tubes) correspond to dye behaviour in: (A) Video clip 4; (B) 902 Video clip 12; and (C) Video clip 13. Large arrow in (A): direction of free-stream flow in (A) $903-(\mathrm{C})$. Do and Me: Streamlines arising from passage through (numbered) sensory channels in 904 the dorsal and medial quadrants of olfactory rosette, respectively (cf. Fig. 7C). EN: Excurrent 905 nostril; Ey: eye; IN: incurrent nostril; V1-3: vortices.

906

907 Fig. 10 Incurrent nostril entry points for CFD-generated streamlines passing through the 908 olfactory sensory channels in the nasal chamber of the Huso dauricus model. Dashed line: 909 contour enclosing region of fluid where speed of flow is $\geq 8 \mathrm{~cm} \mathrm{~s}^{-1}$. White regions: areas through which (A) lateral (Lt), (B) dorsal (Do), (C) medial (Me), (D) ventral (Ve), and (E) all

911 'sensory channel' streamlines pass. Scale bar in (E): 0.25 mm. d: Dorsal; 1: lateral; m: medial; 912 v: ventral.

913

914 Fig. 11 Static pressure on the surface of Huso dauricus CFD model. Surface colour-coded 915 according to pressure coefficient ( $C p$, panel B). (A) Anterior aspect of head. (B) Anterior and 916 (C) lateral aspect of nasal region. Cross in (A) and (B), arrow in (C): direction of free-stream 917 flow (into page for cross). Disks: points of relatively high static pressure. White line in (C): 918 main division between $\mathrm{C} p>0$ (red) and $\mathrm{C} p<0$ (blue). Ba: Barbel; $\mathrm{CB}$ : position of cartilaginous 
919 bar; EN: excurrent nostril; Ey; eye; IN: incurrent nostril; NR: nasal region; Ro: rostral tip; W:

920 incurrent nostril wall.

922 Fig. 12 CFD-generated streamlines in the nasal chamber of the Huso dauricus model. Single streamlines (yellow tubes) passing through the sensory channels of the (A) lateral (Lt), (B) dorsal (Do), (C) medial (Me), and (D) ventral (Ve) quadrants of the right olfactory rosette. For clarity, not all of the streamlines passing through the dorsal and medial sensory channels are shown. Sensory channels marking the limits of streamline groups are numbered, and highlighted by disks, as are other sensory channels of note. Olfactory rosette colour-coded according to pressure coefficient ( $C p$, panel B). Dashed lines in (B) and (C): streamlines passing through (lateral) sensory channel 3 and (ventral) sensory channel 23, respectively.

930 Scale bar in (D) also applies to (A) - (C). a: Anterior; CS: central support; EN and IN: position 931 of excurrent and incurrent nostril, respectively; La: olfactory lamella; p: posterior; SC: sensory 932 channel; V1, V2, and V4 (white streamline tube): vortices.

934 Fig. 13 CFD-generated streamlines showing flow decelerating as it approaches the central 935 support of the olfactory rosette of the Huso dauricus model. Compare with Video clip 5 and 936 Fig. 8E. Arrows, asterisk, and black on white lines: direction of flow, central support, and positions of incurrent (IN) and excurrent nostrils (EN), respectively. Streamlines colour-coded according to speed $(U)$. La: Olfactory lamella; SC: sensory channel.

940 Fig. 14 Static pressure on the surface of Pseudoscaphirhynchus kaufmanni CFD model. 941 Surface colour-coded according to pressure coefficient ( $C p$, panel C). (A) Anterior and (B) 942 lateral aspect of head. Arrow: direction of free-stream flow. Yellow dot: location of probe used 943 to monitor static pressure in transient CFD simulation. Inset in (B): magnified view of nasal 944 region (slightly different orientation to main image). $\alpha$ : angle at which lateral wall of incurrent 945 nostril inclined to body axis. White line: main division between $\mathrm{C} p>0$ (red) and $\mathrm{C} p<0$ (blue). 946 (C) Anterior aspect of nasal region. Inset: anterolateral view of nasal region. Disks: points of 947 relatively high static pressure. Cross: free-stream flow into page. White asterisks: eye; yellow 948 asterisk: olfactory rosette's central support. CB: Position of cartilaginous bar; $C p$ : pressure 949 coefficient; EN: excurrent nostril; IN: incurrent nostril; NR: nasal region; Ro: rostral tip; Sp: 950 spines (Vecsei and Peterson, 2004); W: incurrent nostril wall.

952 Fig. 15 Nasal regions of Acipenser schrenckii (BMNH 1925.8.6.3) and Scaphirhynchus 
954 view of right nasal region of A. schrenckii. (D) Anterior view of right nasal region of $S$. 955 platorynchus. All images flipped horizontally. CS: Central support; EN: excurrent nostril; Ey: 956 eye; IN: incurrent nostril; NF: nasal flap; Sp: spine; W: incurrent nostril wall.

957

958 Fig. 16 CFD-generated streamlines in the (translucent) left nasal region of the 959 Pseudoscaphirhynchus kaufmanni model. Dorsal aspect. Compare with Video clip 15. Arrow: 960 direction of free-stream flow. Upper inset: enlargement (rotated and horizontally flipped - see 961 triangular marker) of bracketed area in main image. Lower inset: dorsal view of left head. Box: 962 location of main image. Scale bar: $2 \mathrm{~cm}$. EN: Excurrent nostril; Ey: eye; IN: incurrent nostril; 963 NC: nasal chamber; V (and asterisk): vortex.

964 
966 Dye visualisation with the plastic sturgeon models. Flow is left to right and the free-stream

967 speed is $5 \mathrm{~cm} \mathrm{~s}^{-1}$. Unless stated otherwise: the model is that of Huso dauricus; pitch, yaw, and 968 roll are $0^{\circ}$; the model is in orientation I, being viewed from flume face X (Fig. 4B); and each 969 clip is flipped horizontally.

970

971 Clip 1 Passage of dye over head and through nasal chamber. Left lateral (slightly posterior) 972 aspect. Compare with Fig. 8A.

973 Clip 2 Passage of dye over head and through nasal chamber. Right nasal region, dorsal aspect. 974 Orientation II, flume face Y (Fig. 4B). Compare with Fig. 8B.

975 Clip 3 Passage of dye through olfactory sensory channels 1 and 24 to 26 (24 - 25 s). Left nasal 976 region, lateral (slightly posterior) aspect. Compare with Fig. 8C.

977 Clip 4 Passage of dye through nasal chamber. Left nasal region, lateral aspect. Compare with 978 Fig. 8D.

979 Clip 5 Fanning of dye in vicinity of central support of olfactory rosette. Right nasal region, 980 lateral aspect. Orientation II (Fig. 4B). Clip rotated by $180^{\circ}$. Compare with Fig. 8E.

981 Clip 6 Passage of dye through sensory channels 1 to 3 (45 - 46 s) and 23 and 24 (43 - 44 s). 982 Right nasal region, anterolateral aspect. Orientation II (Fig. 4B). Clip rotated $180^{\circ}$. Compare 983 with Fig. 8F.

984 Clip 7 Passage of dye through sensory channels 1 and 22 to 24 (54 - 56 s). Right nasal region, 985 anterolateral aspect. Pitch $-5^{\circ}$, flume face Z (Fig. 4B). Clip not flipped or rotated. Compare 986 with Fig. 8G.

987 Clip 8 Passage of dye through sensory channels 20 to 23 ( $1 \mathrm{~min} 7 \mathrm{~s}$ to $1 \mathrm{~min} 8 \mathrm{~s}$ ). Right nasal 988 region, lateral aspect. Pitch - $5^{\circ}$, flume face Z (Fig. 4B). Clip not flipped or rotated. Compare 989 with Fig. 8H.

990 Clip 9 Passage of dye through sensory channels 3 to 5 ( 1 min 13 s to 1 min 15 s). Left nasal 991 region, lateral aspect. Compare with Fig. 8I.

992 Clip 10 Passage of dye through sensory channels 1 and 2 ( $1 \mathrm{~min} 18 \mathrm{~s}$ to $1 \min 20 \mathrm{~s}$ ), 17 and 18 993 (1 min $20 \mathrm{~s}$ onwards) and 24 to 26 (1 min $21 \mathrm{~s}$ ). Left nasal region, anterolateral aspect. Pitch + $994 \quad 5^{\circ}$. Compare with Fig. 8J.

995 Clip 11 Passage of dye through sensory channels 4, 5 (1 min $30 \mathrm{~s})$ and 6 (1 $\mathrm{min} 31 \mathrm{~s}$ to $1 \mathrm{~min}$ $99632 \mathrm{~s}$ ). Right nasal region, lateroventral aspect. Orientation III, roll + 34 ${ }^{\circ}$ (Fig. 4B). Clip rotated $997180^{\circ}$. Compare with Fig. 8K.

998 Clip 12 Vortices in nasal chamber. Left nasal region, lateral aspect. Compare with Fig. 8L.

999 Clip 13 Vortex in nasal chamber. Left nasal region, lateral aspect. Compare with Fig. 8M. 
1000 Clip 14 Passage of dye through (translucent) nasal chamber of plastic model of 1001 Pseudoscaphirhynchus kaufmanni. Left nasal region, lateral aspect. Orientation III, roll + 90 , 1002 flume face Y (Fig. 4B). Clip not flipped or rotated.

1003 Clip 15 Dye behaviour in (translucent) nasal chamber of plastic model of 1004 Pseudoscaphirhynchus kaufmanni. Left nasal region, dorsal aspect. Orientation III, roll - 90 1005 (Fig. 4B). Clip rotated 180. Compare with Fig. 16.

1006 Clip 16 Separation of flow from back of head. Left laterodorsal aspect. 


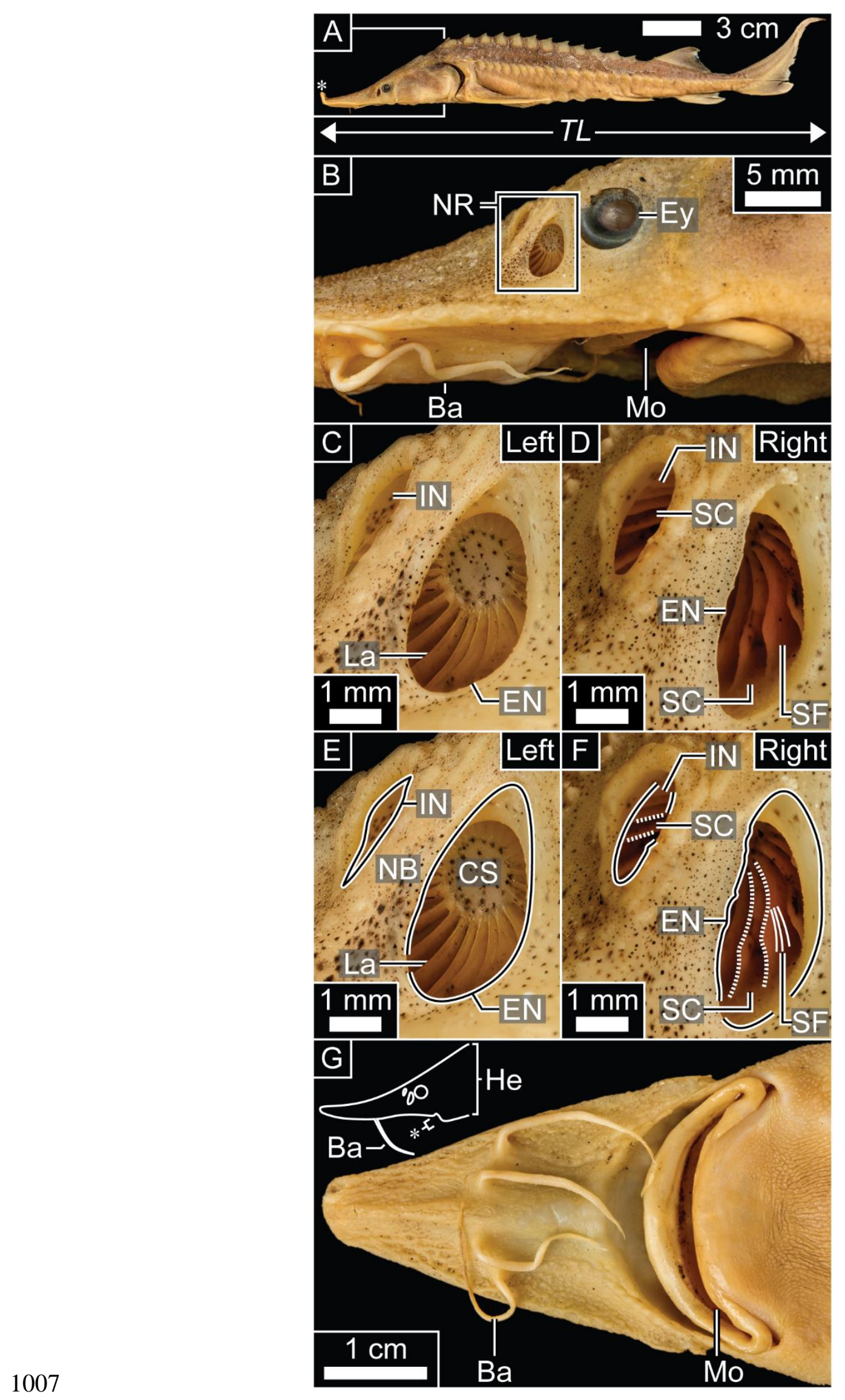

1009 Fig. 1 Specimen of Huso dauricus (BMNH 1925.8.6.2) used to generate the models for 1010 fluid dynamics. 
1011
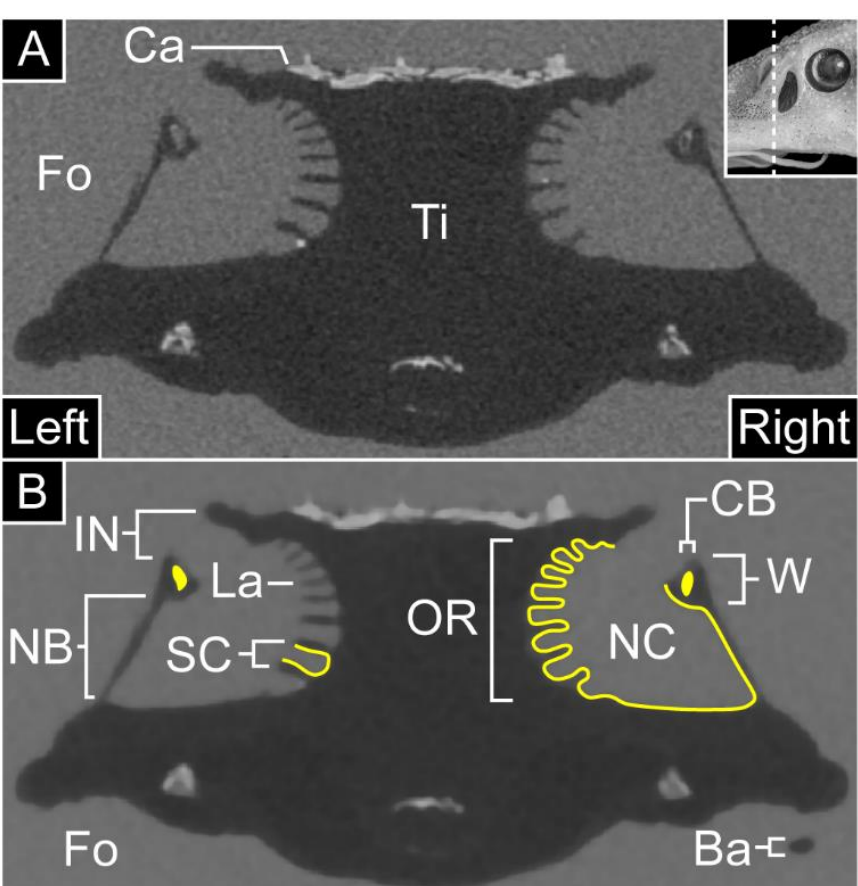

1012

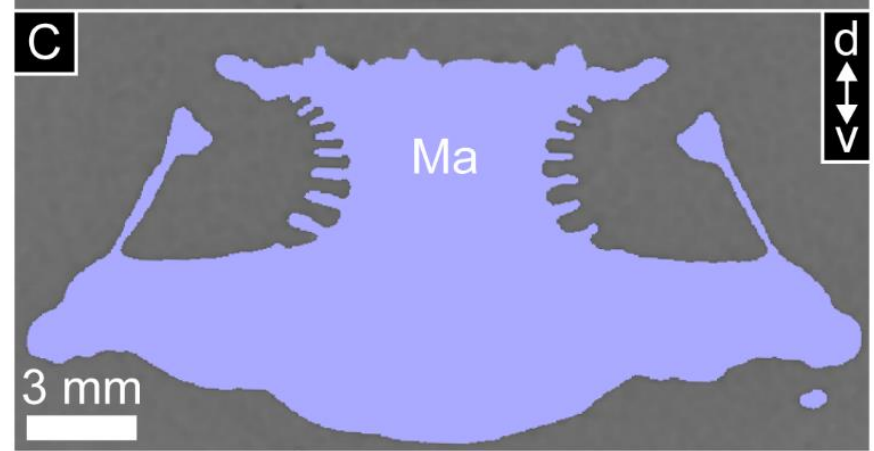

1013

1014 Fig. 2 Image processing. 


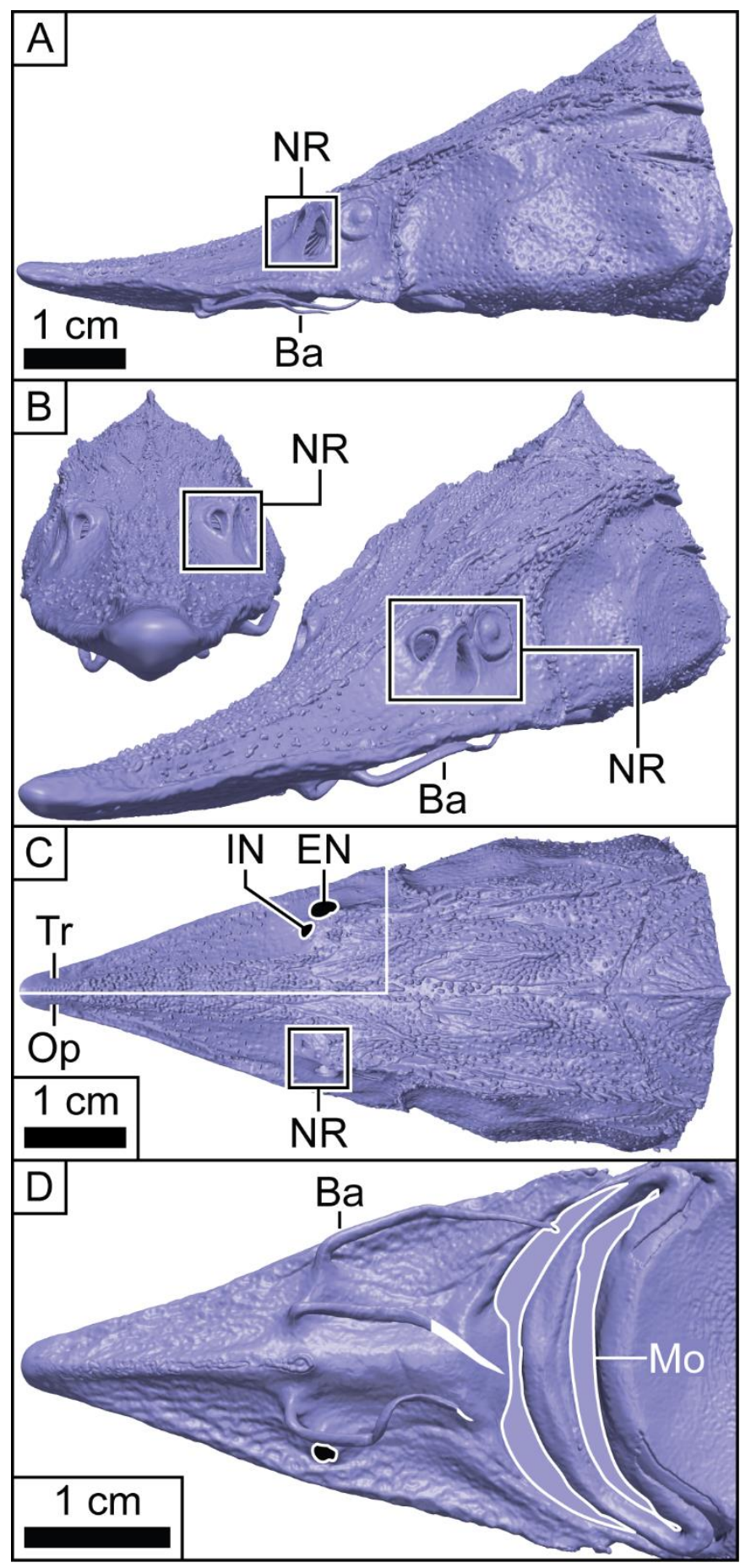

1015

1016

1017 Fig. 3 Surface model of Huso dauricus. 


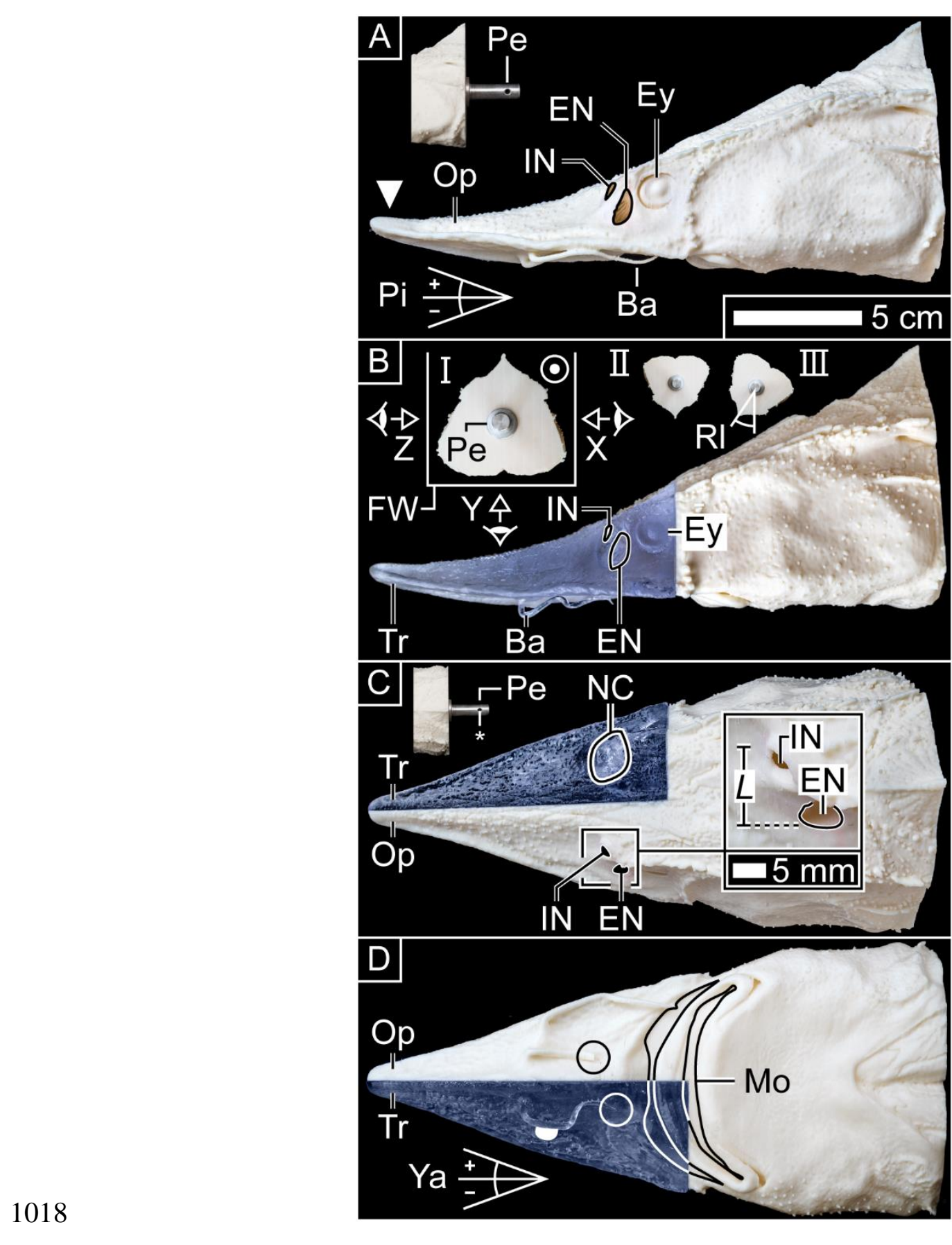

1019

1020 Fig. 4 Plastic model of Huso dauricus. 


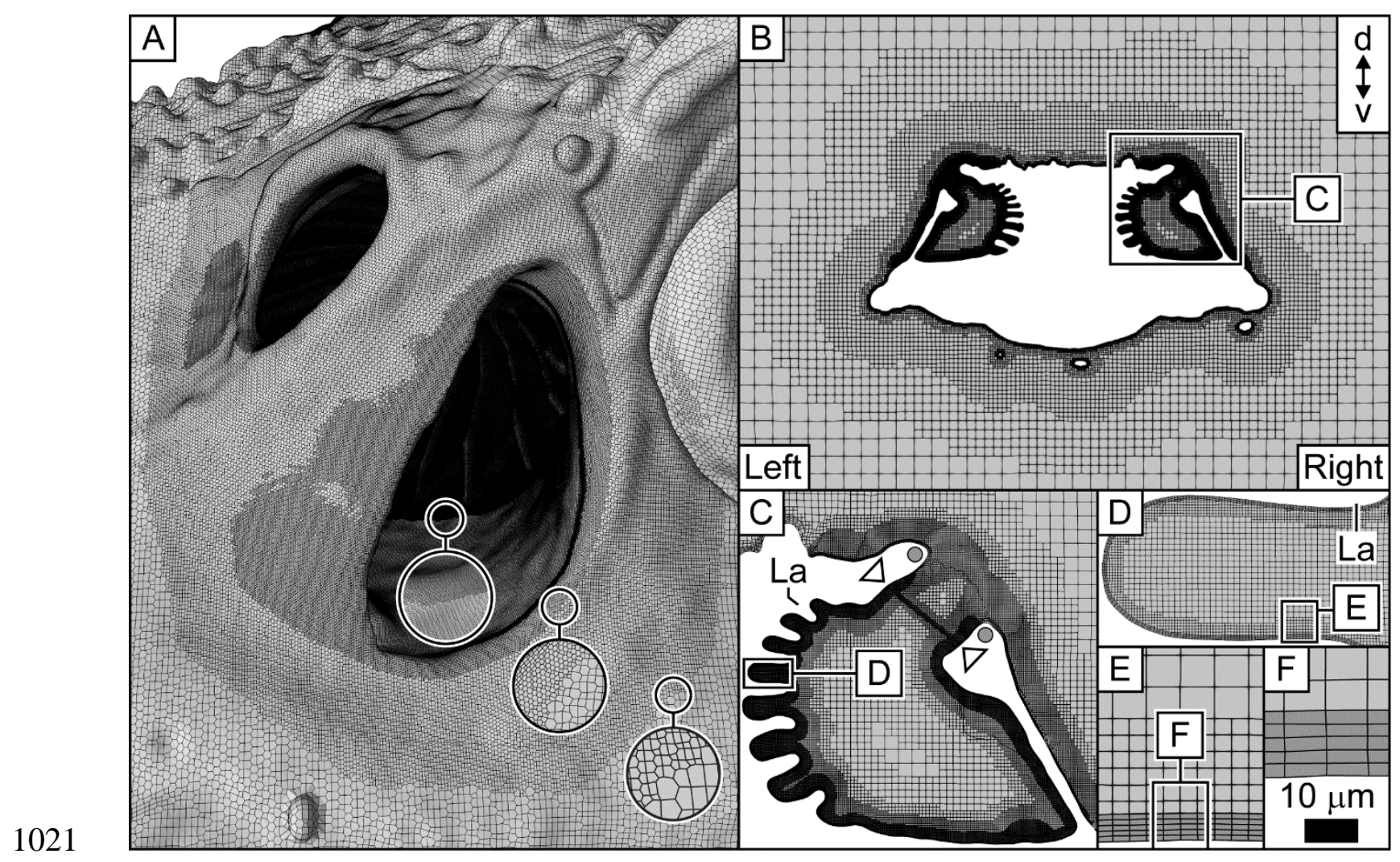

1022

1023 Fig. 5 CFD mesh of Huso dauricus. 


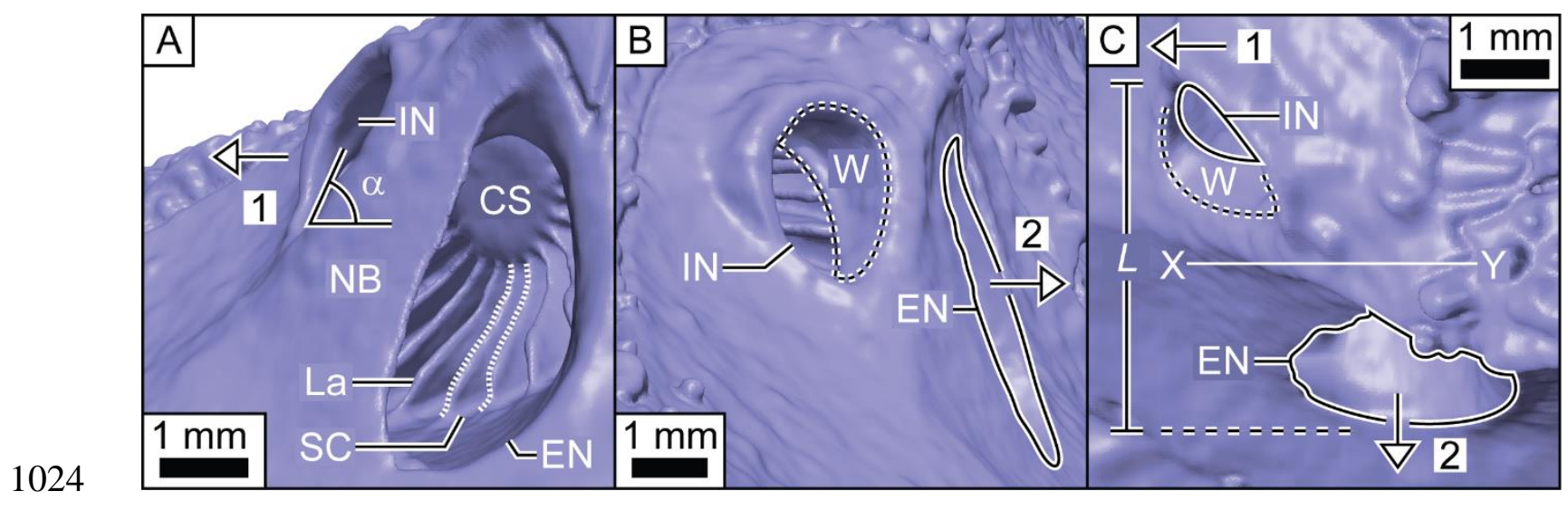

1025

1026 Fig. 6 Nasal region of Huso dauricus surface model (corresponding to the right nasal 1027 region of specimen). 


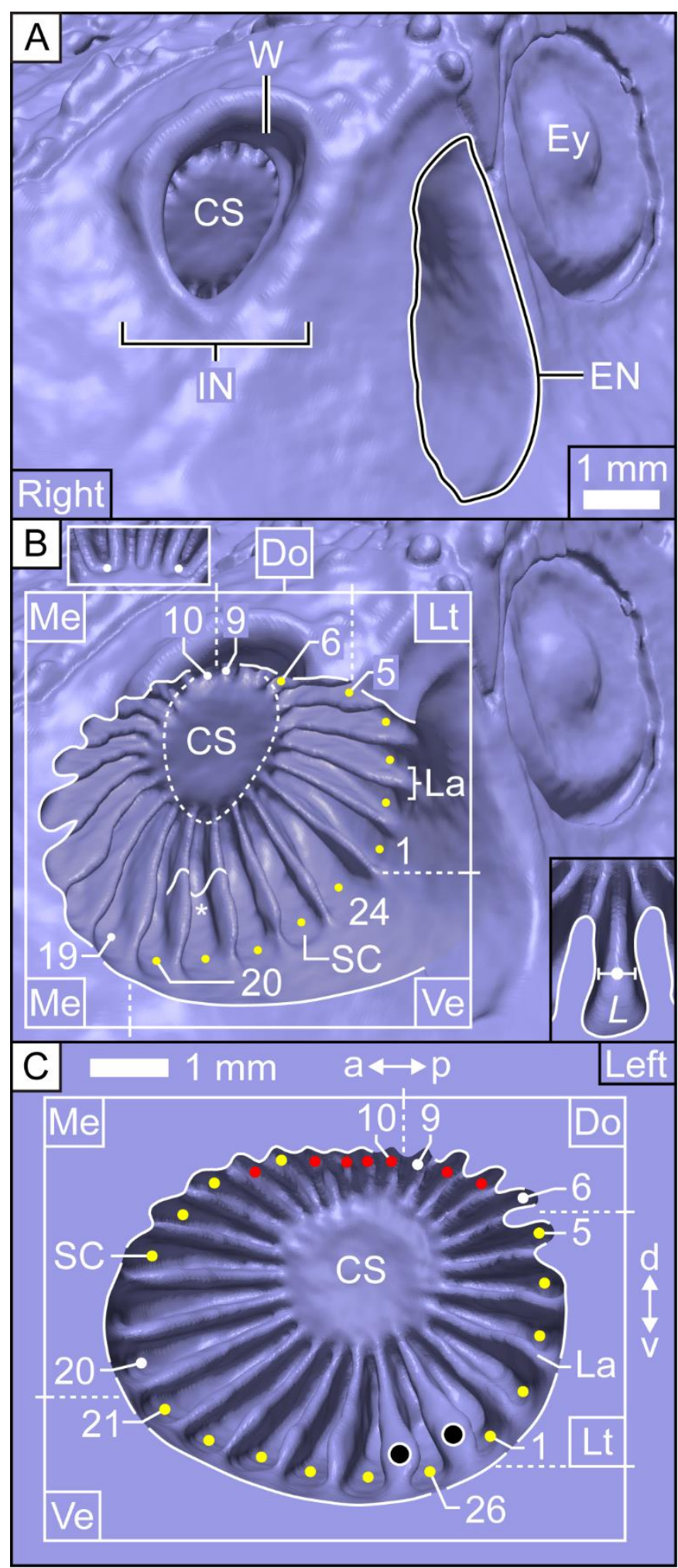

1029

1030 Fig. 7 Olfactory rosettes of Huso dauricus surface model. 


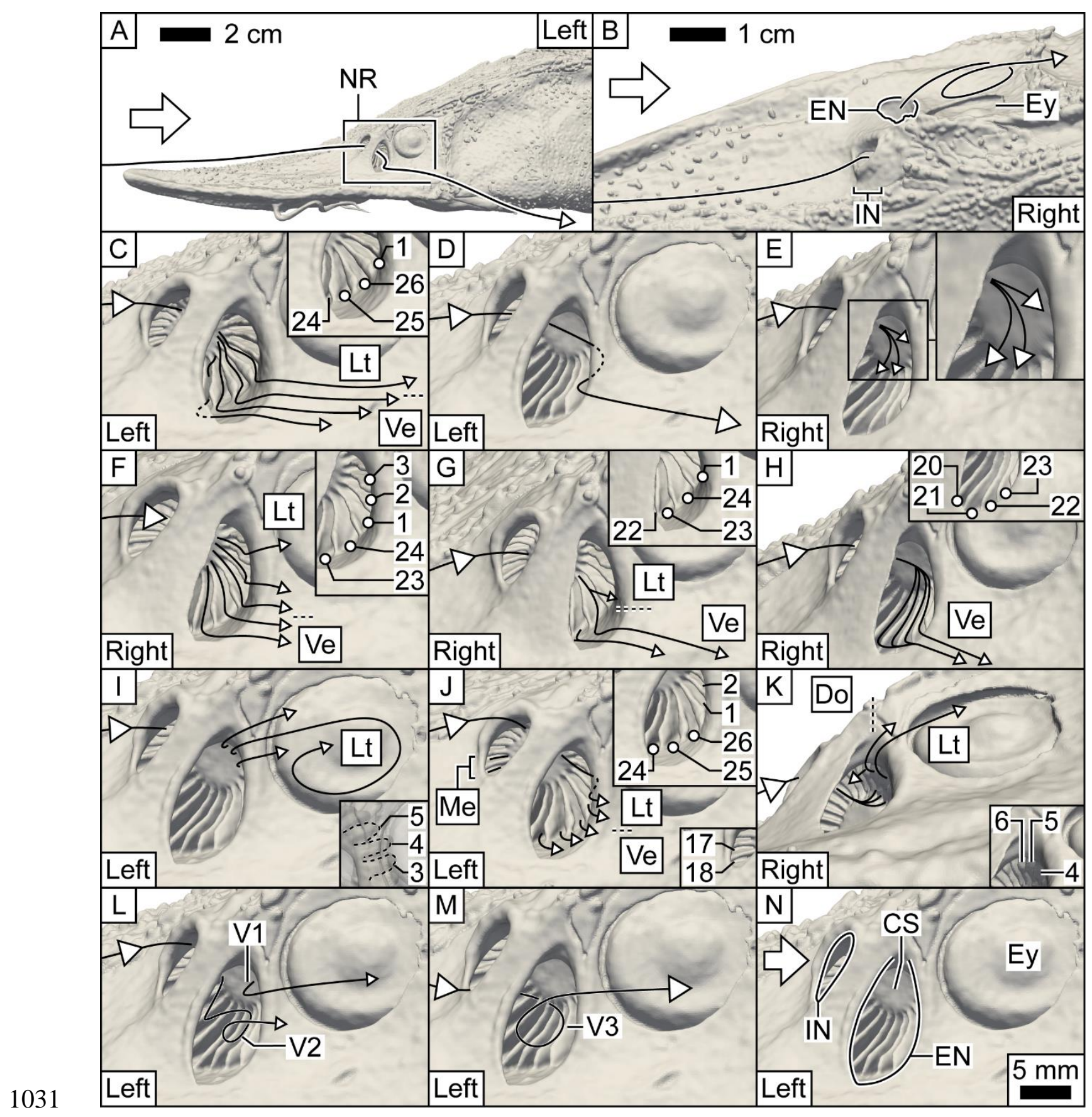

1032

1033 Fig. 8 Schematics of olfactory flow in the plastic model of Huso dauricus. 1034 


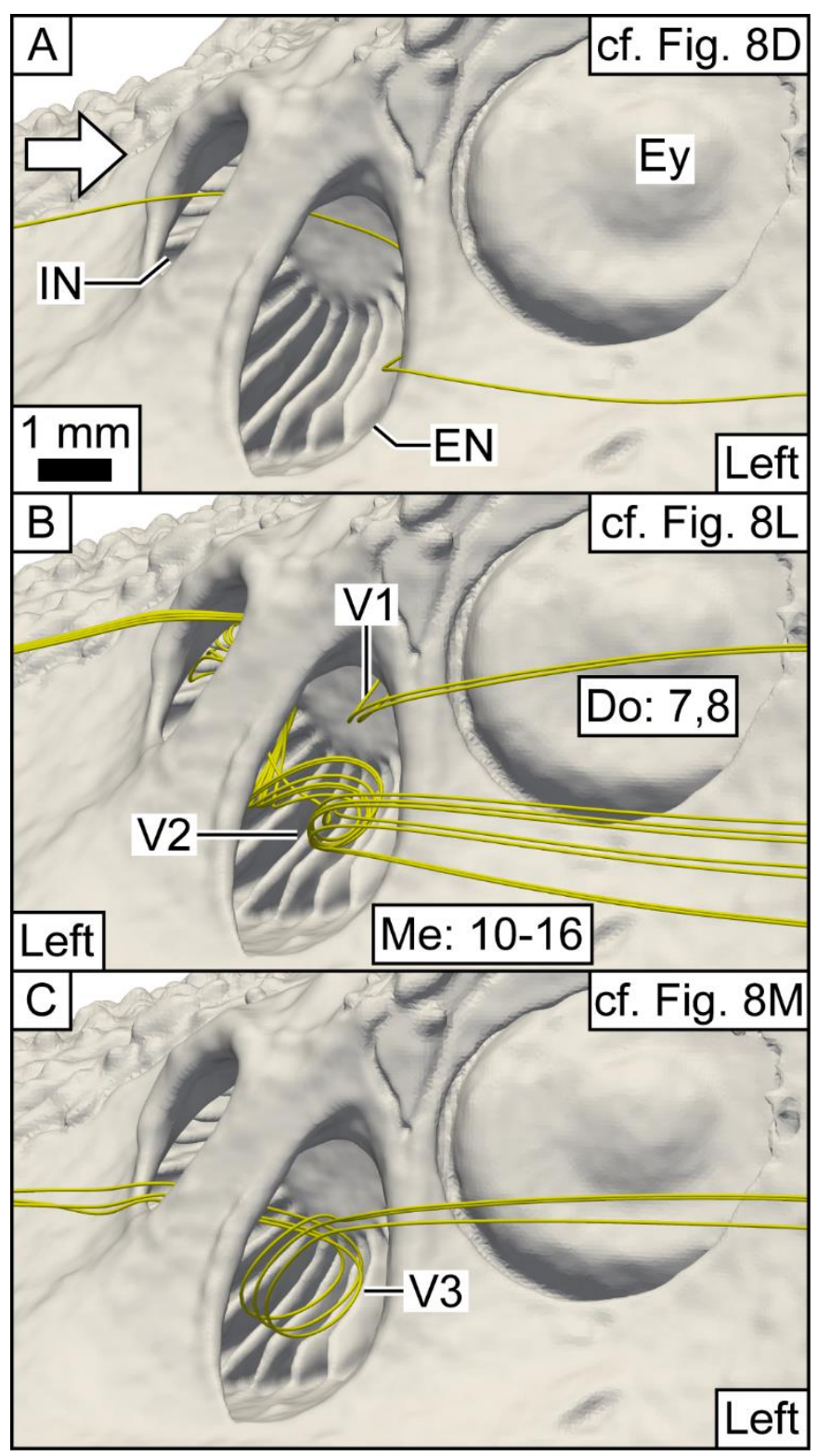

1037 Fig. 9 Correspondence of CFD-generated streamlines to olfactory flow in the plastic 1038 model of Huso dauricus. 


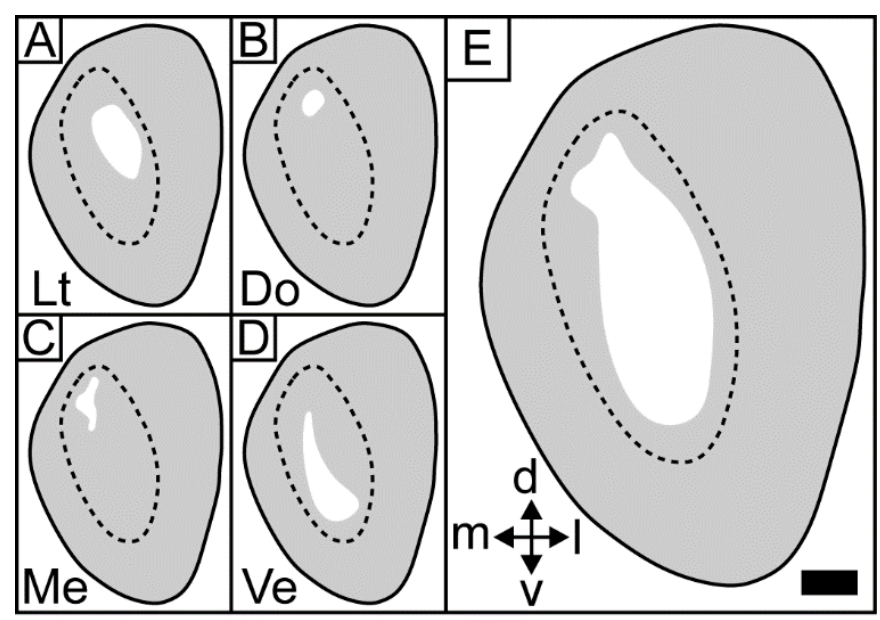

1042

1043 Fig. 10 Incurrent nostril entry points for CFD-generated streamlines passing through

1044 the olfactory sensory channels in the nasal chamber of the Huso dauricus model. 


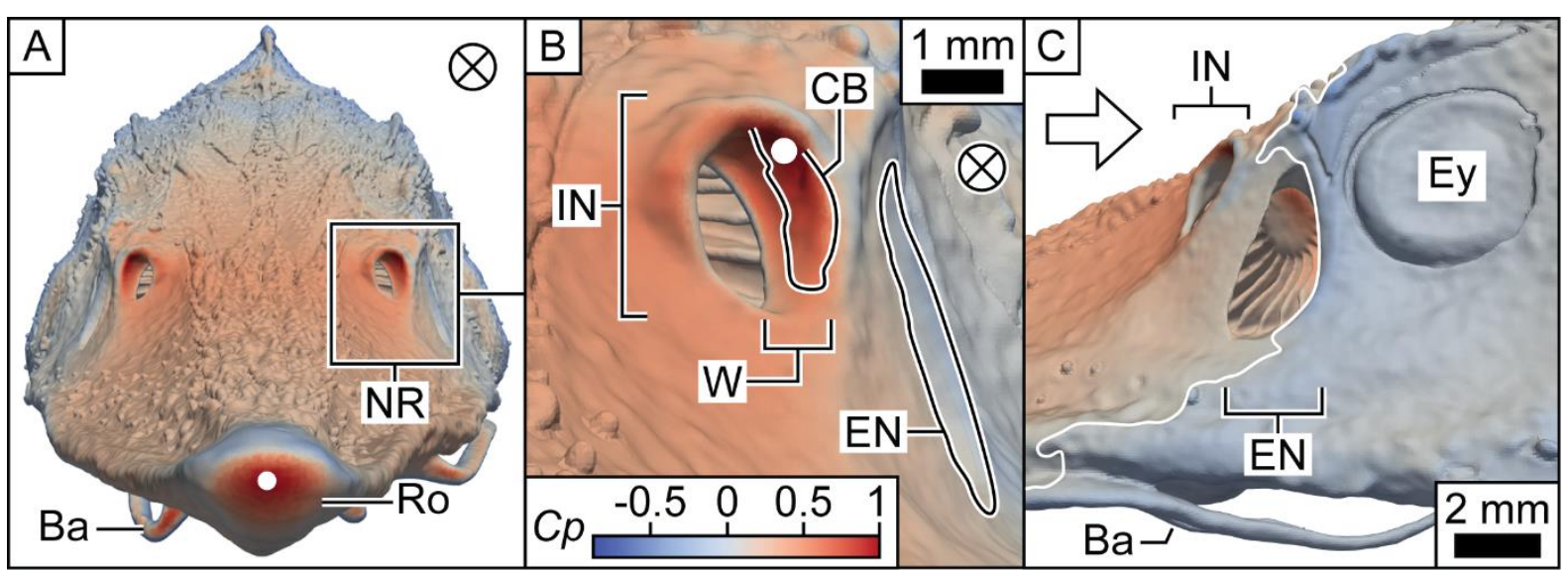

1048

1049 Fig. 11 Static pressure on the surface of Huso dauricus CFD model. 


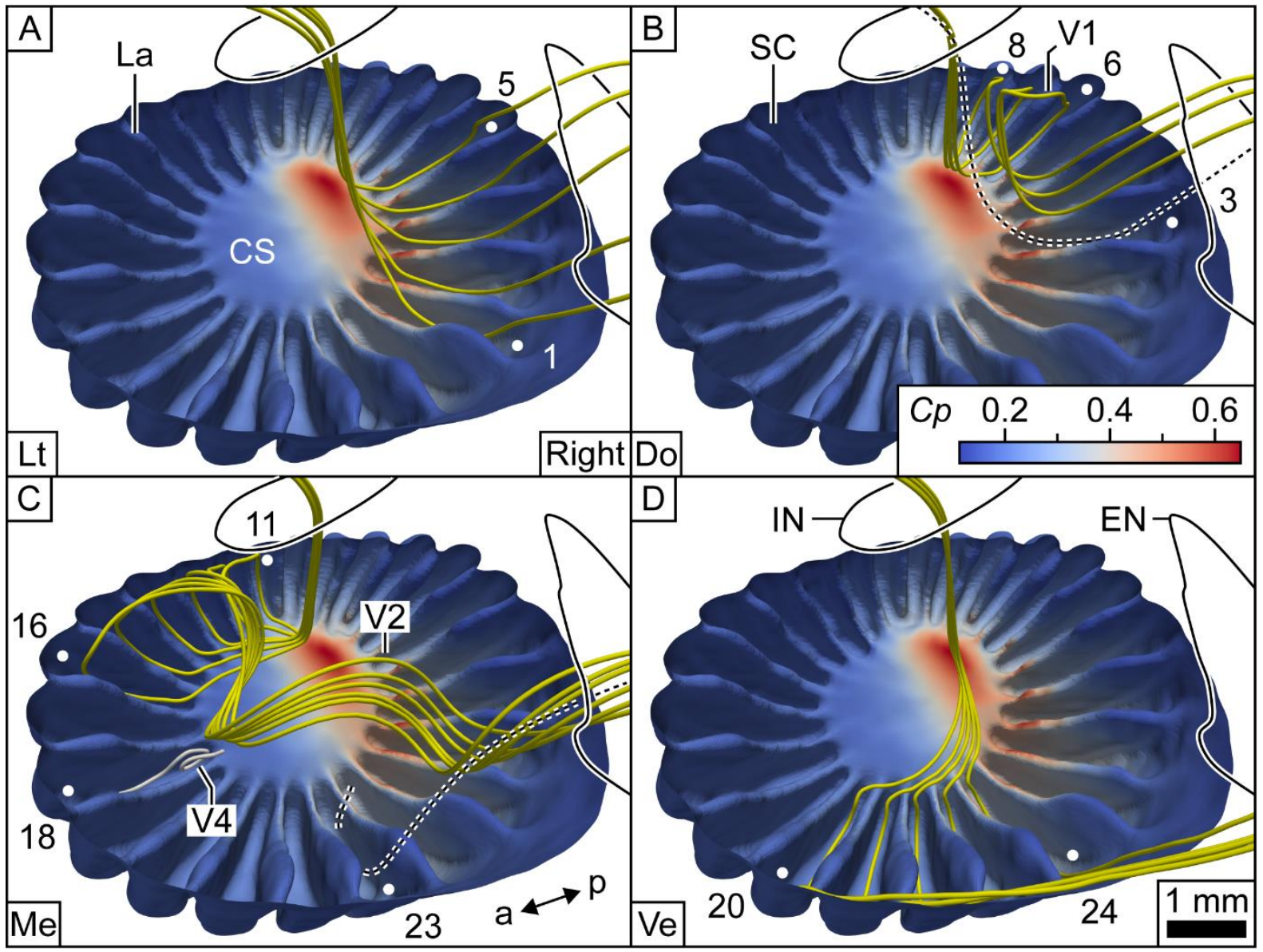

Fig. 12 CFD-generated streamlines in the nasal chamber of Huso dauricus model. 
1056

1057

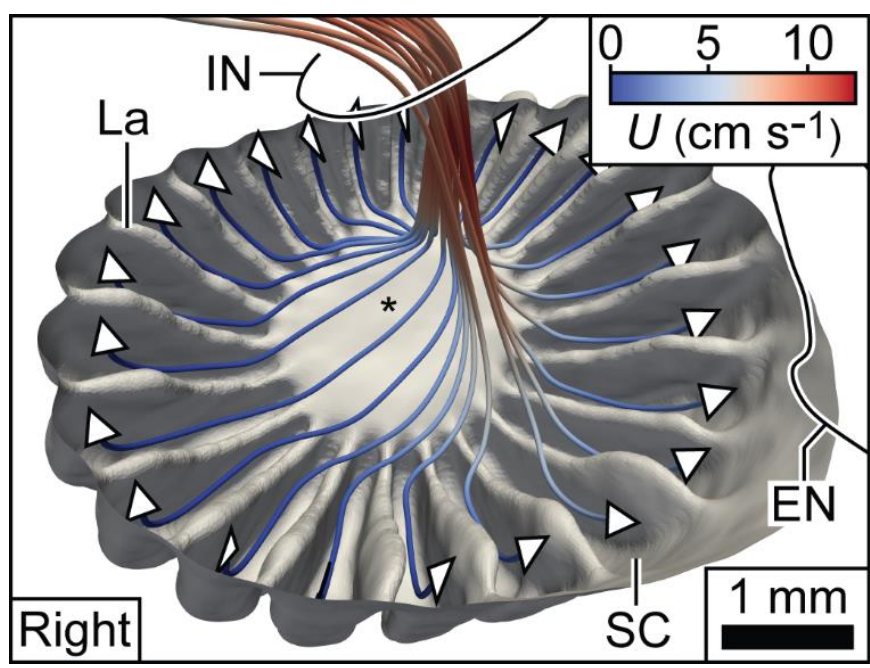

1058

1059 Fig. 13 CFD-generated streamlines showing flow decelerating as it approaches the central 1060 support of the olfactory rosette of the Huso dauricus model. 


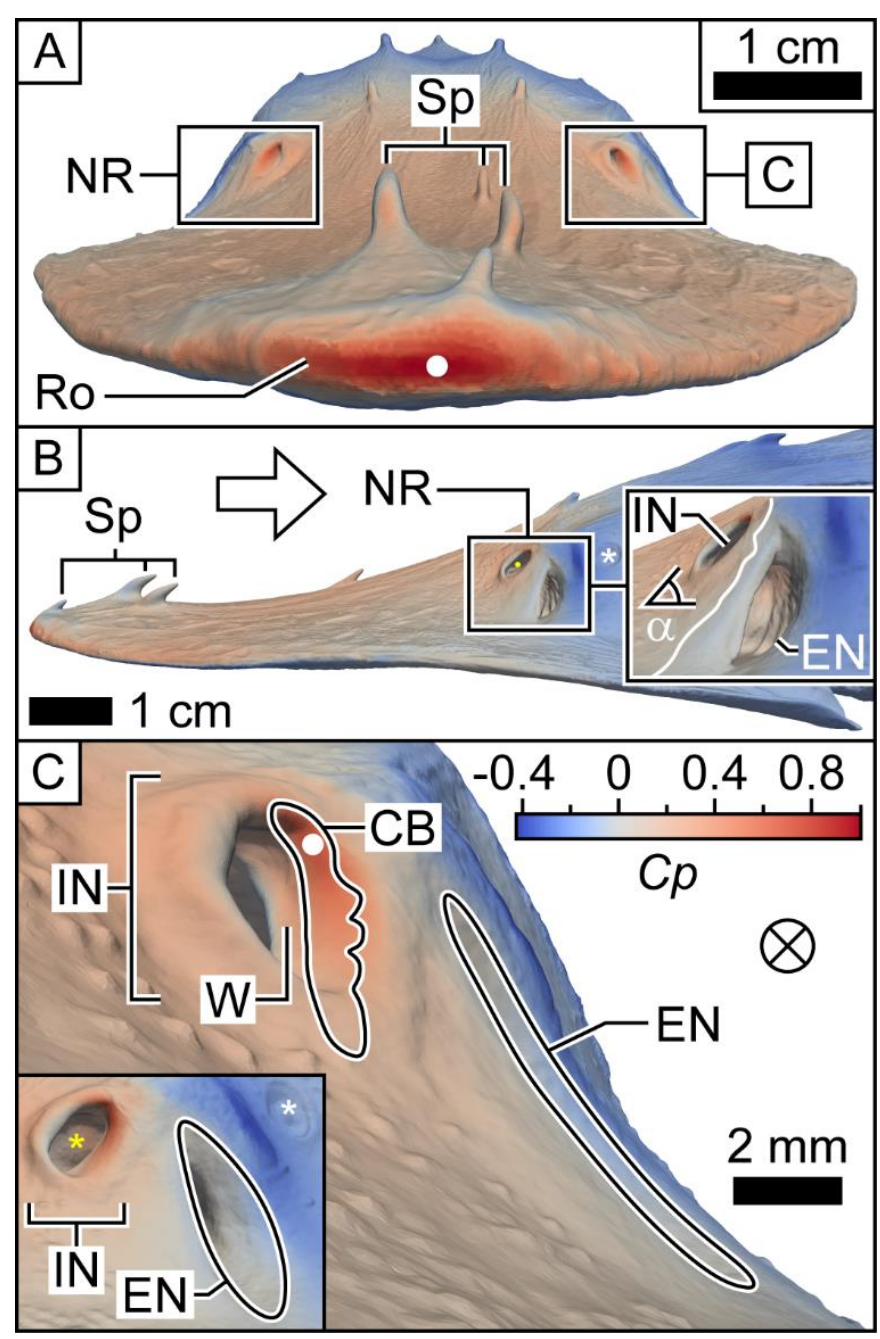

1064

1065 Fig. 14 Static pressure on the surface of Pseudoscaphirhynchus kaufmanni CFD model. 1066 


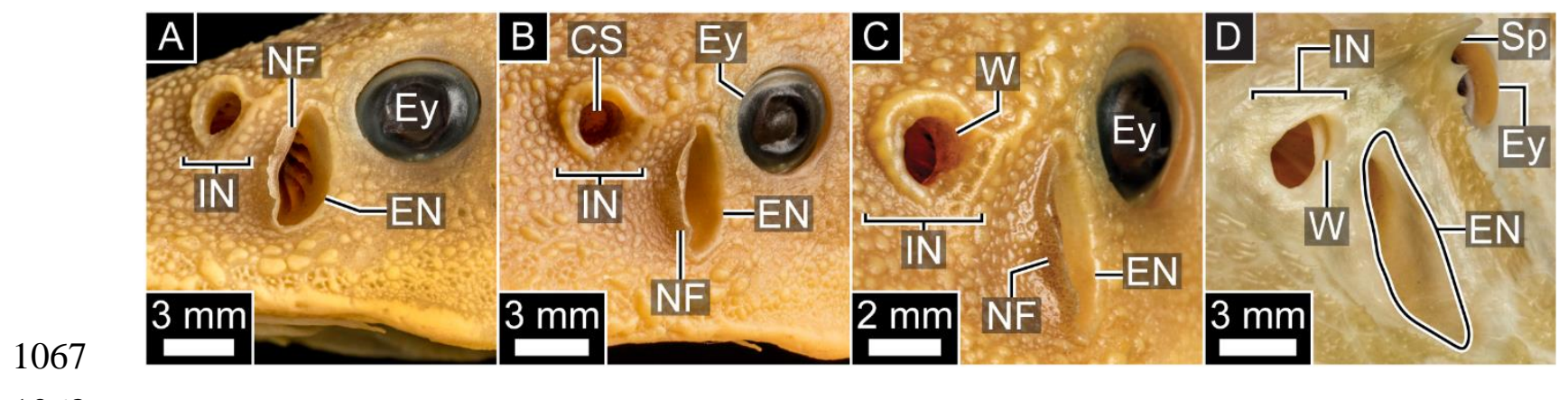

1068

1069 Fig. 15 Nasal regions of Acipenser schrenckii (BMNH 1925.8.6.3) and Scaphirhynchus 1070 platorynchus (BMNH 1852.8.16.26) specimens.

1071 


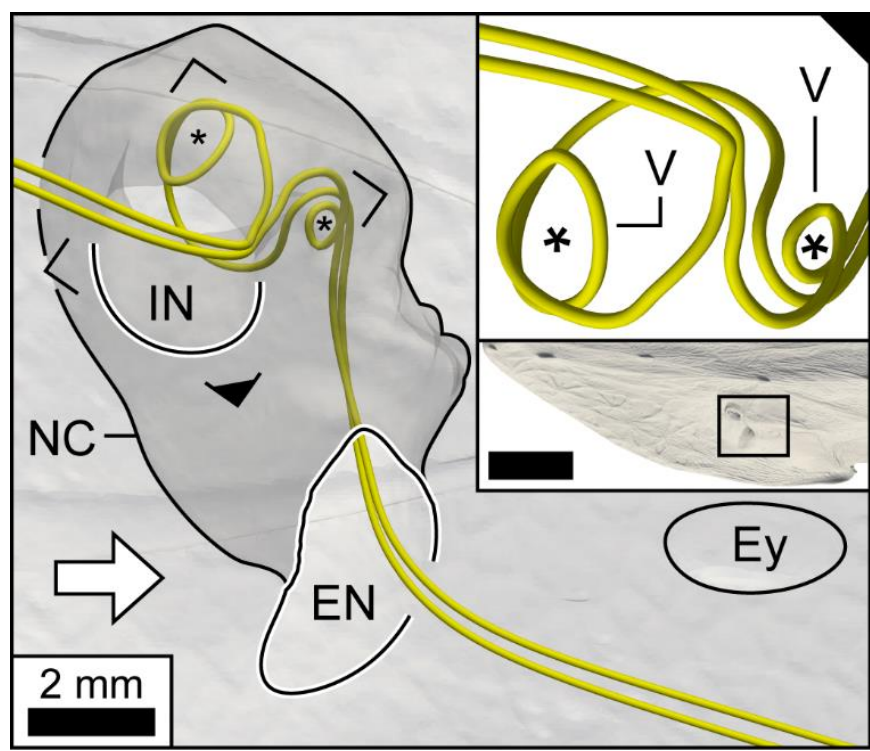

1073

1074 Fig. 16 CFD-generated streamlines in the (translucent) left nasal region of the 1075 Pseudoscaphirhynchus kaufmanni model.

1076 
1079 Russell J. Garwood ${ }^{\mathrm{a}}$, Julia Behnsen ${ }^{\mathrm{b}}$, Harriet Haysom ${ }^{\mathrm{c}}$, Jeremy N. Hunt ${ }^{\mathrm{d}}$, Luke J. Dalby ${ }^{\mathrm{e}}$,

1080 Samuel K. Quilter ${ }^{\mathrm{e}}$, James S. Maclaine ${ }^{\mathrm{f}}$, Jonathan P. L. Cox ${ }^{\mathrm{c}^{*}}$

1081

$1082{ }^{a}$ School of Earth and Environmental Sciences, University of Manchester, Manchester, M13

1083 9PL, $U K$

$1084{ }^{b}$ Henry Moseley X-ray Imaging Facility, University of Manchester, Manchester, M13 9PY,

$1085 U K$

$1086{ }^{c}$ Department of Chemistry, University of Bath, Bath, BA2 7AY, UK

1087 dJeremy Hunt Design, Unit A6, 66 Norlington Road, London, E10 6LA, UK

$1088{ }^{e}$ TotalSim, Top Station Road, Brackley, NN13 7UG, UK

$1089{ }^{f}$ Department of Life Sciences, Natural History Museum, Cromwell Road, London, SW7 5BD,

$1090 \quad U K$

1091

1092

$1093 *$ Corresponding author

1094 Dr Jonathan P.L. Cox

1095 Department of Chemistry

1096 University of Bath

1097 Bath, BA2 7AY, UK

1098 Tel. +441225386548

1099 j.p.1.cox@bath.ac.uk 


\section{A.1. Additional methodology}

1103

\section{A.1.1. X-ray micro-computed tomography}

1105 The specimen of Huso dauricus was held in a plastic cylinder during the micro-CT scan, with 1106 the body axis of the specimen vertical and the head up. To prevent the specimen moving, a 1107 strip of muslin was wrapped around the body. The X-ray beam was generated from a static 1108 tungsten reflection target and passed through a $0.25 \mathrm{~mm}$ copper filter. Exposure time (single 1109 image), accelerating voltage, and current were $708 \mathrm{~ms}, 145 \mathrm{kV}$, and $85 \mu \mathrm{A}$, respectively. A 1110 total of 3142 projections were collected in a single $360^{\circ}$ rotation at $0.114577^{\circ}$ intervals. The 1111 projections were transformed into a 3D matrix using CT Pro 3D 2.2 (Nikon Metrology). Prior 1112 to converting the scan to a set of 8-bit TIFFs, the contrast between pixels corresponding to 1113 the tissue of the specimen and those corresponding to Fomblin was improved (using Drishti)

1114 by making a non-linear adjustment to the histogram of greyscale values.

\section{A.1.2. Surface models}

1117 TIFF images from the micro-CT scan of H. dauricus were segmented with ScanIP's

1118 Threshold tool. To avoid capturing pixels corresponding to Fomblin, which had greyscale 1119 values lying between those of the pixels corresponding to the tissue and those corresponding 1120 to the cartilaginous skeleton (Fig. 2A), 'tissue' and 'cartilage' pixels were captured separately 1121 by the thresholding process, creating 'tissue' and 'cartilage' masks. The tissue and cartilage 1122 masks were then joined with the Boolean operations tool (Union with) to create a mask of the 1123 complete head ('head' mask). To facilitate the joining process, prior to the Boolean Union 1124 operation a Morphological filter (Dilate) was applied to the cartilage mask, using a

1125 Structuring element (ball) with a radius of one pixel in the $\mathrm{x}, \mathrm{y}$, and $\mathrm{z}$ directions. The 1126 Floodfill, Paint, and 3D editing (Cuboid) tools were used to remove the barbel, to seal the 1127 mouth, to put a hole in the back of the 'opaque' mask (below) for the plastic model's 1128 aluminium peg (Fig. 4A-C, Pe), and to fill internal cavities (to reduce the size of the STL file 1129 prior to 3D printing/conversion to the CFD mesh). For the reason explained in Appendix 1130 A.1.3, the head mask was reflected through its median plane (Evans, 1993, p. xi) with the 1131 Flip tool. The size of the head mask was adjusted with the Rescale tool. To make the plastic 1132 model, two new masks were created, one ('opaque' mask) corresponding to the plastic 1133 model's opaque part (Fig. 4A), the other ('translucent' mask) to the translucent part (Fig. 4B). 
1134 The translucent mask was isolated from the head mask with the Floodfill tool. The opaque

1135 mask was generated with the Boolean operations tool by subtracting the translucent mask

1136 from the head mask. A surface model was created from each mask with the following

1137 features (de)selected in ScanIP's 'Model configuration' dialogue box: a) 'General' tab $\rightarrow$

1138 Smart mask smoothing (pre-processing) $\rightarrow$ Use greyscale values; b) 'Surface settings' tab $\rightarrow$

1139 Triangle smoothing $\rightarrow$ Use triangle smoothing for masks (10 iterations); and c) 'Surface

1140 settings' tab $\rightarrow$ Decimation $\rightarrow$ Decimate box unticked.

\section{$1142 \quad$ A.1.3. Plastic model}

1143 We considered the left nasal region of the $H$. dauricus specimen to be more suitable for dye

1144 visualisation than the right, because the anterior edge of the left excurrent nostril appeared

1145 more robust and smoother than its right counterpart (cf. Fig. 1C and D). Consequently the left

1146 nasal region was chosen to become the translucent part of the plastic model. This meant,

1147 however, that the model would be upside down when observed from the flume's most

1148 convenient viewing face (Fig. 4B, face X). Consequently, to return the model to its upright

1149 state, the surface model was, during its preparation, reflected through its median plane

1150 (Appendix A.1.2). Thus the plastic model's (opaque) left nasal region (Fig. 4A) corresponds

1151 to the specimen's right nasal region, and the plastic model's (translucent) right nasal region

1152 (Fig. 4B) corresponds to the specimen's left nasal region. Similarly, the CFD model's left

1153 nasal region corresponds to the specimen's right nasal region, and the CFD model's right

1154 nasal region corresponds to the specimen's left nasal region.

1156 A.1.4. Fluid dynamics

1157

1158 A.1.4.1. Dye visualisation

1159 The plastic model of $H$. dauricus was suspended in the flume using the rig described in Abel

1160 et al. (2010). The model was fixed to the rig via its aluminium peg (Fig. 4A-C, Pe). One of

1161 the holes in the peg (Fig. 4C, asterisk) allowed the model to be upright or upside down

1162 (orientations I and II, Fig. 4B). The rig/peg arrangement also allowed the pitch, yaw, and roll

1163 (Barnard and Philpott, 2004) of the model to be varied (Fig. 4A, B and D). The model was

1164 positioned such that it was central $( \pm 2 \mathrm{~cm})$ width-wise to the working section of the flume.

1165 The maximum transverse cross-sectional area of the model was $61 \mathrm{~cm}^{2}$, less than $5 \%$ of the

1166 working cross-sectional area of the flume. Therefore, based on standard corrections (Barlow

1167 et al., 1999, p. 361), the effect of the walls of the flume on flow in the vicinity of the model is

1168 likely to have been negligible. The model was illuminated with a halogen lamp or an LED 
1169 light panel. A white sheet was placed behind the model to help visualise dye. The dye

1170 solution was introduced from a reservoir under constant pressure using stainless steel tubing

1171 (internal diameter $1.3 \mathrm{~mm}$, external diameter $2.0 \mathrm{~mm}$ ). The horizontal section of this tubing,

1172 from which dye was released, was $25 \mathrm{~cm}$ from the flume's floor. At a free-stream speed of 5

$1173 \mathrm{~cm} \mathrm{~s}^{-1}$, dye emerged from the tubing as a well-defined filament, indicating that the exit

1174 velocity of the dye was equal to the local flow velocity (Fig. 3.1 of Lim, 2000); at free-stream

1175 speeds $>5 \mathrm{~cm} \mathrm{~s}^{-1}$, however, the dye filament became turbulent. To minimise the effect of the

1176 tubing on flow over the model, the aperture of the tubing was located some distance (10 - 11

$1177 \mathrm{~cm}$ ) upstream from the point of impingement on the model (Lim, 2000). We observed dye

1178 behaviour in the nasal chamber mainly through the excurrent nostril (e.g. Fig. 8C), but also

1179 through the incurrent nostril (Fig. 8J) and through the translucent plastic corresponding to the

1180 specimen's left nasal region (Fig. 8I).

1181

1182 A.1.4.2. Computational fluid dynamics

1183 The tail (Fig. A.2) was added to the back of the H. dauricus STL model with GeoMagic

1184 Wrap, essentially as follows. The posterior surface of the model was replicated (Polygons $\rightarrow$

1185 Boundaries $\rightarrow$ Create $\rightarrow$ Boundary By Crease Angle $\left[18^{\circ}\right]$ ) and offset caudally (Polygons $\rightarrow$

1186 Offset $\rightarrow$ Offset Entire Model [23.2 cm]). The posterior edge of the model was extruded

1187 (Polygons $\rightarrow$ Boundaries $\rightarrow$ Move $\rightarrow$ Extrude Boundary $[2 \mathrm{~cm}]$ ), and then joined to the

1188 offset posterior surface with a series of $\sim 50$ bridging surfaces (Polygons $\rightarrow$ Fill Holes $\rightarrow$ Fill

1189 Single $\rightarrow$ Bridge $\rightarrow$ Flat), and the gaps between the bridging surfaces filled (e.g. Polygons $\rightarrow$

1190 Fill Holes $\rightarrow$ Fill Single $\rightarrow$ Bridge $\rightarrow$ Curvature). Prior to joining, the offset posterior surface

1191 was reduced in size (Tools $\rightarrow$ Transform $\rightarrow$ Scale). The scaled offset surface, together with

1192 the offset distance, gave the tail a taper angle of $7^{\circ}$ (Fig. A.2), which from previous CFD

1193 simulations was known to prevent flow separating from the back of a model. The tail was

1194 smoothed (e.g. Polygons $\rightarrow$ Smooth $\rightarrow$ Relax) and imperfections in the model removed

1195 (Polygons $\rightarrow$ Mesh Doctor, e.g. with Self-Intersections and Small Tunnels boxes ticked).

1196

1197 Points of relatively high static pressure on the surface of the $H$. dauricus CFD model were

1198 located using ParaView's Find Data tool.

1200 The average static pressure in the excurrent nostril was calculated in ParaView by first using 1201 the Slice filter to put through the mesh a plane that passed through the excurrent nostril, and 1202 then applying to that plane the following succession of filters: Connectivity $\rightarrow$ Threshold (to 
1203 isolate the segment of the plane within the excurrent nostril) $\rightarrow$ Calculator (to calculate the

1204 static pressures at all points within this segment) $\rightarrow$ Integrate Variables. The average pressure

1205 was then found by dividing the 'pressure' entry (Attribute: Point Data) in the Spreadsheet

1206 view by the Area entry (Attribute: Cell Data).

1208 Streamlines were generated by applying the Stream Tracer With Custom Source filter to

1209 either a point or line, with the following menu selections (selections in brackets): Vectors

1210 (velocity); Interpolator Type (Interpolator with Point Locator); Integration Direction (Both);

1211 Integrator Type (Runge-Kutta 4.5); Integration Step Unit (Cell Length); Initial Step Length

1212 (0.2 m); Minimum Step Length (0.01 m); Maximum Step Length (0.5 m); Maximum Steps

1213 (2000); Maximum Streamline Length (0.2 m); Terminal Speed (10-12 $\left.\mathrm{m} \mathrm{s}^{-1}\right)$; Maximum Error

$1214\left(10^{-6}\right)$. The point or line was created from the Sources menu (Point Source or Line).

1215

1216 To designate the white areas shown in the insets of Fig. 10, a line of five equally spaced

1217 points was first placed along the length of each sensory channel. A streamline was generated

1218 from each point. The white areas were then designated with the Clip filter, using a plane that

1219 passed through the nostril and the relevant group of streamlines.

\section{A.1.5. Nasal chamber volumes}

1222 The volume of each nasal chamber of the H. dauricus model was calculated using ScanIP, as

1223 follows. The Floodfill tool was used to create from the head mask a mask corresponding to

1224 the Fomblin. The 3D editing (Cuboid) tool was then used to cut the Fomlin mask flush to

1225 both the incurrent and excurrent nostril apertures, and the nasal chamber volume isolated with

1226 the Floodfill tool. This volume was then converted to a surface model as described in

1227 Appendix A.1.2. The volume of the nasal chamber surface model was read from the Model

1228 Statistics tab.

1230 A.1.6. Reynolds numbers for olfactory flow through nasal chamber (CFD simulation)

1231 The volumetric flow rate through each nasal chamber of $H$. dauricus was determined in

1232 ParaView by first using the Slice filter to put through the mesh a plane that passed through

1233 the nasal chamber (Fig. 6C, line XY) and then applying the following succession of filters:

1234 Connectivity $\rightarrow$ Threshold (to isolate the segment of the plane within the nasal chamber) $\rightarrow$

1235 Surface Vectors (to select velocity vectors perpendicular to the nasal chamber segment) $\rightarrow$

1236 Calculator (to determine the magnitude of the velocity vectors) $\rightarrow$ Integrate Variables. The

1237 wetted perimeter of the nasal chamber was estimated from a perpendicular view of the 
1238 segment by importing the view into Rhinoceros, tracing the outline of the segment with the

1239 Free-Form command (Curve $\rightarrow$ Free-Form $\rightarrow$ Control Points), and then calculating the

1240 length of the line with the Length command (Analyze $\rightarrow$ Length).

1241

1242 A.2. Effect of reflecting Huso dauricus model

1243 Given the symmetrical fluid environment for both the dye visualisation experiments and the

1244 CFD simulation (Section 2.5), we assumed that reflecting the model of $H$. dauricus

1245 (Appendix A.1.2) would not affect olfactory flow. We tested this assumption by observing

1246 dye behaviour in the opaque nasal region (Fig. 4A) when the model was both upright and

1247 upside down in the flume (orientations I and II, Fig. 4B). Dye behaved similarly in both cases

1248 (cf. Video clip 5, orientation II, with Video clip 8, orientation I). Furthermore, flow behaved

1249 similarly in both nasal regions despite their mild asymmetry (Section 3.1). Therefore

1250 reflecting the model had little or no effect on olfactory flow.

1251

1252 A.3. Additional references

1253 Barlow, J.B., Rae, W.H., Pope, A., 1999. Low-Speed Wind Tunnel Testing. 3rd ed. John

1254 Wiley \& Sons, New York.

1255 Barnard, R.H., Philpott, D.R., 2004. Aircraft Flight. 3rd ed. Pearson Education, Harlow.

1256 Evans, H.E., 1993. Miller's Anatomy of the Dog. $3^{\text {rd }}$ ed. W.B. Saunders Company,

1257 Philadelphia. 

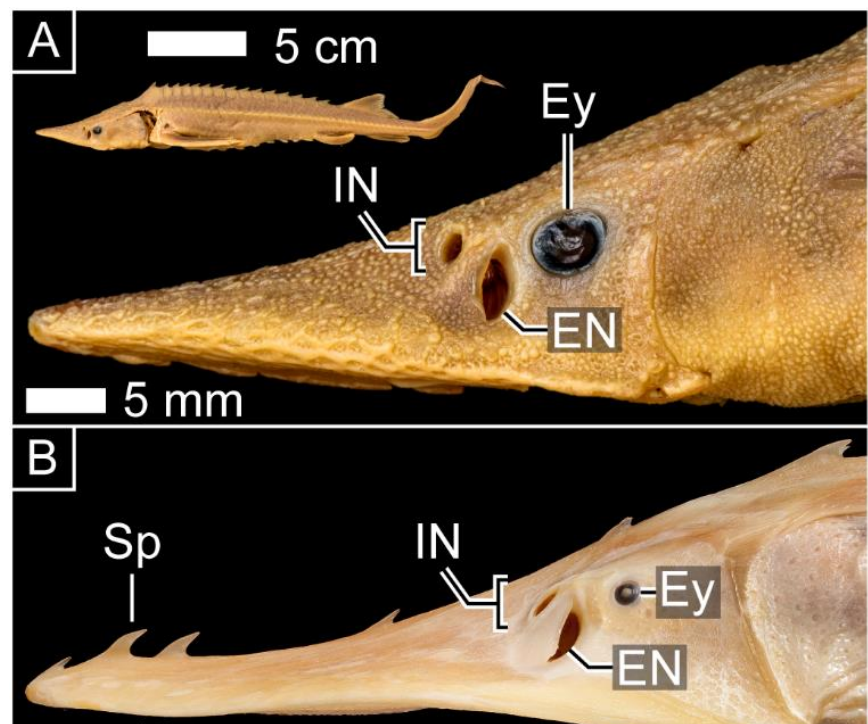

1262 Fig. A.1. Heads of other sturgeon species inspected in this study. (A) Acipenser schrenckii

1263 (BMNH 1925.8.6.3). Entire specimen shown above main image. (B) Pseudoscaphirhynchus

1264 kaufmanni (BMNH 1887.4.5.17). Image flipped horizontally. (C) Scaphirhynchus

1265 platorynchus (BMNH 1852.8.16.26). Image flipped horizontally. EN: Excurrent nostril; Ey:

1266 eye; IN: incurrent nostril; Sp: spine.

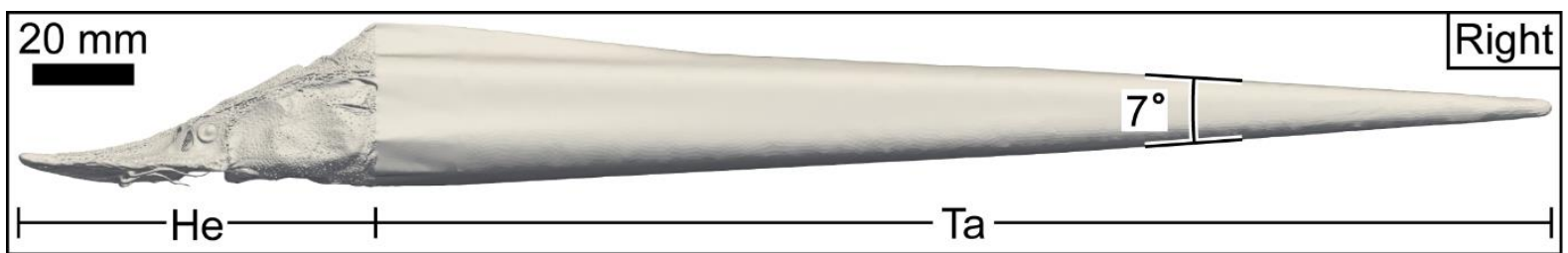

1272 Fig. A.2. CFD model of Huso dauricus. He: Head; Ta: tapered extension ('tail'). 
1274 A.6. Variation in static pressure in transient CFD simulation of Pseudoscaphirhynchus

1275 kaufmanni model

1276

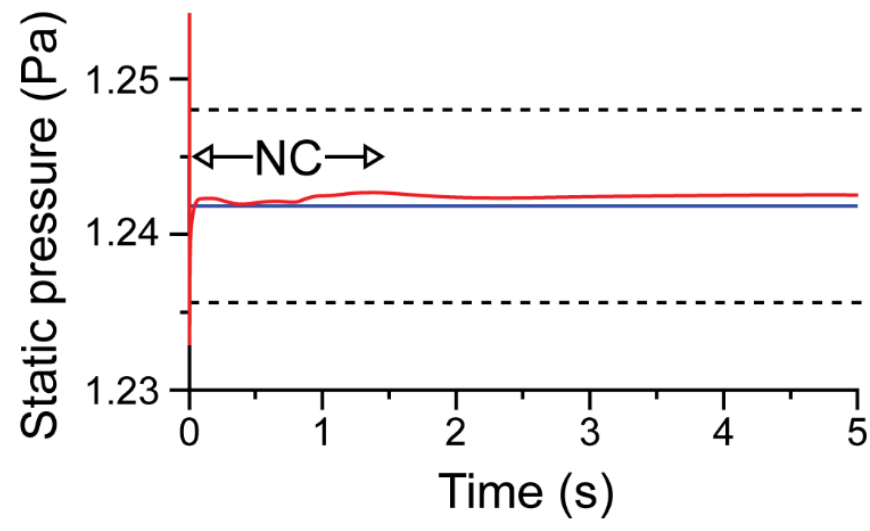

1279 Fig. A.3. Variation in static pressure in transient CFD simulation of Pseudoscaphirhynchus

1280 kaufmanni model. Static pressure monitored in centre of left incurrent nostril (Fig. 14B,

1281 yellow dot). Red line: variation in static pressure. Blue line: average static pressure in last

1282500 iterations of steady-state simulation (Section 2.5.2). Dotted lines indicate $0.5 \%$ deviation

1283 from this average. NC: time taken for nasal chamber to be flushed once in steady-state

1284 simulation. Maximum subsequent deviation between red and blue lines $=0.07 \%$. Simulation

1285 conditions identical to those for Huso dauricus model (Section 2.5.2). 